\title{
Ionization at a solid-water interface in an applied electric field: Charge regulation
}

\author{
Ryuichi Okamoto ${ }^{1}$ and Akira Onuki ${ }^{2}$ \\ ${ }^{1}$ Department of chemistry, Tokyo Metropolitan University, Hachioji, Tokyo 192-0397, Japan \\ ${ }^{2}$ Department of Physics, Kyoto University, Kyoto 606-8502, Japan
}

(Dated: April 19, 2022)

\begin{abstract}
We investigate ionization at a solid-water interface in applied electric field. We attach an electrode to a dielectric film bearing silanol or carboxyl groups with an areal density $\Gamma_{0}$, where the degree of dissociation $\alpha$ is determined by the proton density in water close to the film. We show how $\alpha$ depends on the density $n_{0}$ of $\mathrm{NaOH}$ in water and the surface charge density $\sigma_{m}$ on the electrode. For $\sigma_{m}>0$, the protons are expelled away from the film, leading to an increase in $\alpha$. In particular, in the range $0<\sigma_{m}<e \Gamma_{0}$, self-regulation occurs to realize $\alpha \cong \sigma_{m} / e \Gamma_{0}$ for $n_{0} \ll n_{c}$, where $n_{c}$ is $0.01 \mathrm{~mol} / \mathrm{L}$ for silica surfaces and is $2 \times 10^{-5} \mathrm{~mol} / \mathrm{L}$ for carboxyl-bearing surfaces. We also examine the charge regulation with decreasing the cell thickness $H$ below the Debye length $\kappa^{-1}$, where a crossover occurs at the Gouy-Chapman length. In particular, when $\sigma_{m} \sim e \Gamma_{0}$ and $H \ll \kappa^{-1}$, the surface charges remain only partially screened by ions, leading to an electric field in the interior.
\end{abstract}

\section{INTRODUCTION}

Numerous papers have been written on various aspects of the electric double layer at a solid-water interface 1 . 4 . However, not enough attention has yet been paid on physics and chemistry of ionizable solid surfaces in contact with an aqueous electrolyte solution. As a wellknown example $e^{1-10}$, silanol groups $\mathrm{SiOH}$ on a silica oxide surface at a density $\Gamma_{0}$ dissociate into $\mathrm{SiO}^{-}$and mobile protons $\mathrm{H}^{+}$. The surface charge density is $-e \Gamma_{0} \alpha$, where $\alpha$ is the degree of dissociation. In this case, $\alpha$ is determined by the proton density $n_{\mathrm{H}}(0)$ in water immediately close to the surface. This $n_{\mathrm{H}}(0)$ is different from the bulk proton density $n_{\mathrm{H}}^{0}$, depending on the solution composition. Also when two ionizable surfaces approach, $\alpha$ and the force between them change at small separation $111+16$. These behaviors are often referred to as charge regulation. We note that similar phenomena with variable charges are ubiquitous in soft matters.

We mention theoretical papers on the charge regulation on solid-water interfaces with salts 14 22. Ninham and Parsegian 15 first presented a model of surface ionization using the mass action law as the boundary condition of the Poisson-Boltzmann (PB) equation, where $\Gamma_{0}$ and the dissociation constant $K_{s}$ are relevant parameters. They determined $\alpha$, the charge distribution, and the electric potential self-consistently. Behrens et al! $17+19$ solved these equations accounting for a potential drop across the Stern layer formed on a solid surface in water. These theories are based on one-dimensional (1D) calculations of the PB solutions for symmetric ionizable surfaces. Behrens et al. also devised a formula for ionization of large colloidal particles.

In this paper, we construct a free energy functional for the ion densities $n_{i}$ and $\alpha$ in applied field, accounting for the chemical reactions in the bulk and on the surface. We then examine the effect of sodium hydroxide $\mathrm{NaOH}$ at a low density $n_{0}$, where the hydroxyl density $n_{\mathrm{OH}}$ and the proton density $n_{\mathrm{H}}$ are related by the dissociation law $n_{\mathrm{OH}} n_{\mathrm{H}}=10^{-14} \mathrm{~mol}^{2} / \mathrm{L}^{2}$ in bulk water 23 . Thus, adding $\mathrm{NaOH}$ serves to decrease $n_{\mathrm{H}}(0)$ and increase $\alpha$.
In experiments, the surface ionization due to deprotonation increased with addition of $\mathrm{OH}^{-}$(with increasing $\mathrm{pH}) \sqrt{14 \mid 24} 27$. We also apply an electric field to the system attaching a planar electrode with a surface charge density $\sigma_{m}$. Then, there arises another kind of charge regulation. We assume that the electric double layer next to the film is determined by the effective density $\sigma_{\text {eff }}=-e \Gamma_{0} \alpha+\sigma_{m}$. Thus, if $\sigma_{m}$ is positive (negative), the cations including the protons tend to be repelled from (attracted to) the film, leading to an increase (a decrease) in $\alpha$. We have $\alpha \rightarrow 0$ for $\sigma_{m}<0$ and $\alpha \rightarrow 1$ for $\sigma_{m}>e \Gamma_{0}$. However, in the range $0<\sigma_{m}<e \Gamma_{0}$, marked self-regulation behavior emeges, where $\alpha$ approaches $\sigma_{m} / e \Gamma_{0}$ and $\sigma_{\text {eff }}$ nearly vanishes. In this effect, the $\mathrm{NaOH}$ density $n_{0}$ needs to be smaller than a characteristic density $n_{c}$, where $n_{c}$ is much larger than the hydroxyl density $10^{-7} \mathrm{~mol} / \mathrm{L}$ in pure water. For small wall separation $H$ and for not small $\sigma_{m}$, screening of the surface charges can only be partial, leading to a negative disjoining pressure $\Pi_{d}$ with large amplitude. For sufficiently large $n_{0}$ with small $H$, screening can also be achieved leading to a large positive $\Pi_{d}$. In this paper, we are in the nonlinear $\mathrm{PB}$ regime with addition of $\mathrm{NaOH}$ only, where the Debye length is longer than the Gouy-Chapman length 28 .

Furthermore, in a mixture solvent, the dissociation on solid surfaces can strongly depend on the local solvent composition $\phi$ as well as the solvation of ions in the bulk 29 . In our previous paper ${ }^{30}$, small variations of $\phi$ around the colloid surfaces induced significant changes in $\alpha$ and the ion distribution. Thus, there can be a strong coupling between $\phi$ and the ionization in mixture solvents, which alters adsorption and wetting on the surfaces and the interaction among the colloidal particles.

In polyelectrolytes and charged gels 31 , the dissociation on polymer chains is governed by the local environments and is highly fluctuating in space and time. This can strongly affect coil-globule transition and phase separation, particularly when a second fluid component (cosolvent) is added to a water-like solvent. A first order phase transition of weak-to-strong ionization was also predicted on ionizable rods in a mixture solvent 34 . In 


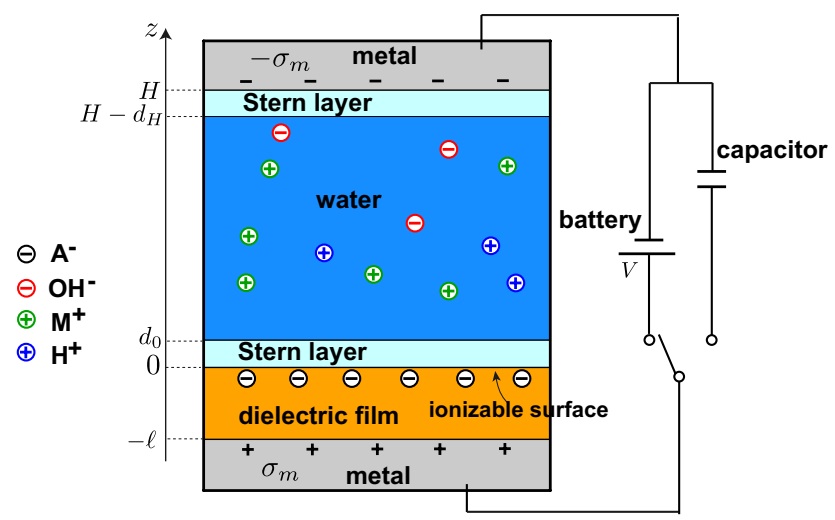

FIG. 1: Illustration of geometry. Two parallel metallic plates are placed at bottom $(z<-\ell)$ and top $(z>H)$ with charge densities $\pm \sigma_{m}$ and a potential difference $V$. A dielectric film with thickness $\ell$ is on the bottom metallic plate $(-\ell<z<0)$. At $z=0$, ionizable groups $\mathrm{AH}$ with a density $\Gamma_{0}$ dissociate into $\mathrm{A}^{-}$and $\mathrm{H}^{+}$with a fraction $\alpha$. Water molecules are under strong influence of the walls in Stern layers $\left(0<z<d_{0}\right.$ and $\left.H-d_{H}<z<H\right)$. Protons also come from dissociation of water. $\mathrm{MOH}$ dissociates into $\mathrm{M}^{+}$and $\mathrm{OH}^{-}$. If a battery is connected, $V$ can be controlled, where the metal surface chage density $\sigma_{m}$ fluctuates. If it is disconnected, $\sigma_{m}$ becomes fixed. Furthermore, if a small capacitor is connected, $\sigma_{m}$ can be changed by a small fixed amount (see Appendix A).

phase separation, $\alpha$ can be very different in the two phases 35 . With addition of alcohol to water, precipitation of DNA has also been observed ${ }^{39}$, where ionization of DNA is favored in water-rich environments.

The organization of this paper is as follows. In Sec.II, we will present a coarse-grained Ginzburg-Landau free energy functional, which includes the electrostatic contributions from the Stern layers and the dielectric film. In Sec.III, we will present numerical results for silicawater interfaces for thick cells. In Sec.IV, we will discuss the ionization on carboxyl-bearing surfaces. In Sec.V, we will present results for thin cells.

\section{THEORETICAL BACKGROUND}

We illustrate our system in Fig.1, where a cell contains liquid water and ions in ambient conditions $(T=300 \mathrm{~K}$ and $p=1 \mathrm{~atm}$ ) in the region $0<z<H$ and a dielectric film is in the region $-\ell<z<0$. For example, we suppose a ultra-thin silica oxide film with silanol groups in water. To apply electric field to the system, we place metallic walls at the two ends in the regions $z<-\ell$ and $z>H$. In our theory, we can fix the applied potential difference $V$ or the electrode surface-change density $\sigma_{m}$.

The cell lengths in the lateral directions are much longer than $H$ such that the edge effect is negligible. All the physical quantities are coarse-grained smooth variables depending only on $z$. Hereafter, the Boltzmann constant will be set equal to 1 .

\section{A. Chemical reactions in bulk and on surface}

In the cell, we initially add a base $\mathrm{MOH}$ at a low density $\sqrt{26}$. It dissociates into mobile univalent cations $\mathrm{M}^{+}$and hydroxide anions $\mathrm{OH}^{-}$as

$$
\mathrm{MOH} \rightleftarrows \mathrm{M}^{+}+\mathrm{OH}^{-} .
$$

In addition, a very small fraction of water molecules dissociate into $\mathrm{H}^{+}$and $\mathrm{OH}^{-}$as 23

$$
\mathrm{H}_{2} \mathrm{O} \rightleftarrows \mathrm{H}^{+}+\mathrm{OH}^{-} .
$$

We use the notation $\mathrm{H}^{+}$, though the protons exist as hydronium ions $\mathrm{H}_{3} \mathrm{O}^{+}$in liquid water. The local number densities of $\mathrm{MOH}, \mathrm{M}^{+}, \mathrm{OH}^{-}$, and $\mathrm{H}^{+}$are written as $n_{\mathrm{MOH}}(z), n_{\mathrm{M}}(z), n_{\mathrm{OH}}(z)$, and $n_{\mathrm{H}}(z)$, respectively, which are coarse-grained smooth functions of $z$ in our theory. The charge density $\rho(z)$ is written as

$$
\rho=e\left(n_{\mathrm{H}}+n_{\mathrm{M}}-n_{\mathrm{OH}}\right) .
$$

In chemical equilibrium, the mass action laws hold:

$$
\begin{aligned}
n_{\mathrm{M}} n_{\mathrm{OH}} / n_{\mathrm{MOH}} & =K_{\mathrm{b}}, \\
n_{\mathrm{OH}} n_{\mathrm{H}} & =K_{\mathrm{w}},
\end{aligned}
$$

where $K_{\mathrm{b}}$ and $K_{\mathrm{w}}$ are the dissociation constants. For $\mathrm{NaOH}$, we have $K_{\mathrm{b}}=10^{-1} \mathrm{~mol} / \mathrm{L}=0.06 \mathrm{~nm}^{-3}$. However, $K_{\mathrm{w}}^{1 / 2}$ is much smaller $\left(=10^{-7} \mathrm{~mol} / \mathrm{L}=6 \times 10^{-8} / \mathrm{nm}^{3}\right)$.

In a thick cell, a homogeneous bulk region appears far from the walls, where $n_{i}$ assume bulk values, written as $n_{i}^{0}$. They satisfy Eqs.(4) and (5) and the charge neutrality condition $n_{\mathrm{M}}^{0}+n_{\mathrm{H}}^{0}=n_{\mathrm{OH}}^{0}$. We introduce the bulk density of $\mathrm{M}$ atoms by

$$
n_{0}=n_{\mathrm{M}}^{0}+n_{\mathrm{MOH}}^{0} .
$$

See Fig.2(a) for $n_{i}^{0}$ vs $n_{0}$ for $\mathrm{M}=\mathrm{Na}$. For such a strong base with large $K_{\mathrm{b}}$, we can well assume $n_{0} \ll K_{\mathrm{b}}$ to find

$$
n_{\mathrm{M}}^{0} \cong n_{0}, \quad n_{\mathrm{MOH}}^{0} \cong n_{\mathrm{OH}}^{0} n_{0} / K_{\mathrm{b}} \ll n_{0},
$$

where $n_{\mathrm{OH}}^{0} \ll K_{\mathrm{b}}$. From $n_{0}=\left(1+n_{\mathrm{OH}}^{0} / K_{\mathrm{b}}\right)\left(n_{\mathrm{OH}}^{0}-n_{\mathrm{H}}^{0}\right)$, we can express $n_{0}$ in terms of $n_{\mathrm{OH}}^{0}$ as

$$
n_{0} \cong n_{\mathrm{OH}}^{0}-n_{\mathrm{H}}^{0}=n_{\mathrm{OH}}^{0}-K_{\mathrm{w}} / n_{\mathrm{OH}}^{0},
$$

In pure water, we have $n_{0}=0$ and $n_{\mathrm{OH}}^{0}=n_{\mathrm{H}}^{0}=K_{\mathrm{w}}^{1 / 2}$. For $n_{0} \gg K_{\mathrm{w}}^{1 / 2}$, we obtain $n_{\mathrm{OH}}^{0} \cong n_{0}$ and $n_{\mathrm{H}}^{0} \cong K_{\mathrm{w}} / n_{0} \ll$ $K_{\mathrm{w}}^{1 / 2}$. In the following figures, supposing a strong base, we will use $n_{0}$ to represent the amount of the added base. However, our theoretical results will be expressed in terms of $n_{\mathrm{OH}}^{0}$ and $n_{\mathrm{H}}^{0}$ and will be valid for arbitrary $K_{\mathrm{b}}$. In our figures Eq.(8) holds, so they can be used even for weak bases 27 if $n_{0}$ is related to $n_{\mathrm{OH}}^{0}$ by Eq. (8)

On the surface of the dielectric film at $z=0$, ionizable groups $\mathrm{AH}$ are distributed with a surface density $\Gamma_{0}$. No 

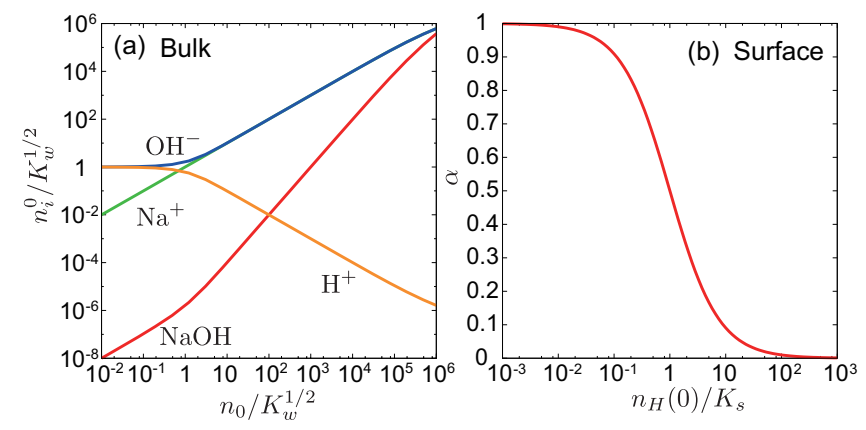

FIG. 2: Chemical reactions in this paper. (a) Equilibrium bulk densities $n_{i}^{0}\left(i=\mathrm{NaOH}, \mathrm{Na}^{+}, \mathrm{OH}^{-}\right.$, and $\left.\mathrm{H}^{+}\right)$divided by $K_{\mathrm{w}}^{1 / 2}$ vs $n_{0} / K_{\mathrm{w}}^{1 / 2}$, where $n_{0}$ is the bulk density of $\mathrm{Na}$ atoms. (b) Equilibrium $\alpha$ vs $n_{\mathrm{H}}(0) / K_{s}$ from Eq.(11).

dissociation is assumed on the upper metal surface. Depending on the local $\mathrm{pH}$ near the surface, a fraction $\alpha$ $(0<\alpha<1)$ of these groups dissociate as ${ }^{15}$

$$
\mathrm{AH} \rightleftarrows \mathrm{A}^{-}+\mathrm{H}^{+} .
$$

Here, $\mathrm{A}^{-}$anions remain on the surface $z=0$, while the dissociated protons are mobile in water. The surface charge density due to $\mathrm{A}^{-}$at $z=0$ is

$$
\sigma_{\mathrm{A}}=-e \Gamma_{0} \alpha .
$$

Let $n_{\mathrm{H}}(0)$ be the proton density immediately close to the dielectric film in water. Then, the surface mass action law in chemical equilibrium is expressed as $\frac{15}{15}$

$$
n_{\mathrm{H}}(0) \alpha /(1-\alpha)=K_{s},
$$

where $K_{s}$ is the surface dissociation constant. See Fig.2b for $\alpha$ vs $n_{\mathrm{H}}(0) / K_{s}$. Since $\alpha=1 /\left[1+n_{\mathrm{H}}(0) / K_{s}\right], \alpha$ tends to 1 (to 0 ) if $n_{\mathrm{H}}(0)$ is much smaller (larger) than $K_{s}$.

We define the particle numbers $N_{i}=\int_{0}^{H} d z n_{i}(z)$ (i= $\mathrm{MOH}, \mathrm{M}, \mathrm{OH}, \mathrm{H})$ per unit area in the cell. Since the hydroxide and proton numbers due to the autoionization coincide, we have

$$
N_{\mathrm{OH}}-N_{\mathrm{M}}=N_{\mathrm{H}}-\Gamma_{0} \alpha .
$$

It follows the overall charge neutrality condition,

$$
\int_{0}^{H} d z \rho(z)+\sigma_{\mathrm{A}}=0 .
$$

In our theory, a reservoir can be attached or the system can be closed. In the latter case, the total number of the $\mathrm{M}$ atoms is fixed as

$$
N_{\mathrm{M}}+N_{\mathrm{MOH}}=H \bar{n},
$$

where $\bar{n}$ is the initial density of the added base.

In Sec.III, we consider silica oxide surfaces with silanol groups $\mathrm{AH}=\mathrm{SiOH}$ and set

$$
\begin{aligned}
K_{s} & =10^{-7.3} \mathrm{~mol} / \mathrm{L}=3.0 \times 10^{-8} / \mathrm{nm}^{3}, \\
\Gamma_{0} & =8.0 / \mathrm{nm}^{2} \quad \text { (silica surface) },
\end{aligned}
$$

where $K_{s}$ is very small $\left(=0.5 K_{\mathrm{w}}^{1 / 2}\right)$. The $\mathrm{pH}$ and $\mathrm{pK}$ values are defined by $n_{\mathrm{H}}^{0}=10^{-\mathrm{pH}} \mathrm{mol} / \mathrm{L}$ (in the bulk region) and $K_{s}=10^{-\mathrm{pK}} \mathrm{mol} / \mathrm{L}$. Then, $\mathrm{pK}=7.3$, $n_{\mathrm{H}}^{0} / K_{s}=10^{7.3-\mathrm{pH}}$, and $n_{\mathrm{OH}}^{0} / K_{s}=10^{\mathrm{pH}-6.7}$ here. The value of $\Gamma_{0}$ in Eq.(15) is obtained for nonporous, fully hydrated silica, so it is large. Experimental values of $\Gamma_{0}$ strongly depend on surface preparation 19 . It is also known that the adsorption $\mathrm{SiOH}+\mathrm{H}^{+} \rightleftarrows \mathrm{SiOH}_{2}^{+}$takes place in high acidity (low $\mathrm{pH}$ ) conditions $\frac{517}{}$, leading to a zero-charge surface state at a pH about 2. See Sec.IV for analysis on ionization on carboxyl-bearing surfaces.

\section{B. Electric potential and Stern layers}

As the electrostatic boundary condition, we may fix the potential difference between the electrodes:

$$
V=\Phi(0)-\Phi(H)+\sigma_{m} / C_{d} .
$$

We may also fix the surface charge density $\sigma_{m}$ on the lower metal surface at $z=-\ell$ (see Appendix A). In the dielectric film, the electric field is given by $4 \pi \sigma_{m} / \varepsilon_{d}$, where $\varepsilon_{d}$ is the film dielectric constant. This yields the potential change $\Phi(-\ell)-\Phi(0)=\sigma_{m} / C_{d}$, where the film capacitance per unit area is written as

$$
C_{d}=\varepsilon_{d} / 4 \pi \ell
$$

In the cell outside microscopic Stern layers (see below), $\Phi(z)$ obeys the Poisson equation,

$$
-\varepsilon_{0} d^{2} \Phi / d z^{2}=4 \pi \rho,
$$

where $\varepsilon_{0}$ is the solvent dielectric constant. The electric field is given by $E=-d \Phi / d z$.

Generally, the electric potential can change noticeably across a microscopic Stern layer at a solid-water interface ${ }^{17340}$. We mention molecular dynamics simulations on this effect 4145 . In our case, there are two such layers at the bottom and top. For simplicity, we assume no specific ion adsorption. Then, the ion amounts in the layers are negligibly small for small bulk ion densities 17 19:43. Denoting the layer thickness as $d_{0}$ at $z=0$ and as $d_{H}$ at $z=H$, we assume linear relations,

$$
\begin{aligned}
V_{0}^{\mathrm{S}} & =\Phi(0)-\Phi\left(d_{0}\right)=\left(\sigma_{m}+\sigma_{\mathrm{A}}\right) / C_{0}, \\
V_{H}^{\mathrm{S}} & =\Phi\left(H-d_{H}\right)-\Phi(H)=\sigma_{m} / C_{H} .
\end{aligned}
$$

For simplicity, the surface capacitances $C_{0}$ and $C_{H}$ are taken to be those in the limit of small ion densities. Here, we can define the polarization $P(z)$ microscopically such that the electric induction $D(z)=E(z)+4 \pi P(z)$ is continuous through the layers $\frac{45}{4}$, where $E(z)$ and $P(z)$ change abruptly in the layers $\$$. We write $D(z)$ at the bottom and the top as

$$
\begin{aligned}
& D\left(d_{0}\right)=\varepsilon_{0} E\left(d_{0}\right)=4 \pi\left(\sigma_{A}+\sigma_{m}\right) . \\
& D\left(H-d_{H}\right)=\varepsilon_{0} E\left(H-d_{H}\right)=4 \pi \sigma_{m},
\end{aligned}
$$


These constitute the boundary conditions of the Poisson equation (18). The effective surface charge density is $\sigma_{\text {eff }}=\sigma_{\mathrm{A}}+\sigma_{m}$ at the bottom. Furthermore, even without applied field $\left(\sigma_{m}=0\right)$, the previous simulations 41 have shown the presence of small potential drops at solid-water interfaces due to the anisotropy of water molecules . We neglect this intrinsic, surface effect in this paper.

The total potential difference $V$ is now written as

$$
V=\left[\Phi\left(d_{0}\right)-\Phi\left(H-d_{H}\right)\right]+\frac{\sigma_{\mathrm{A}}+\sigma_{m}}{C_{0}}+\frac{\sigma_{m}}{C^{\prime}},
$$

where the first term arises from the mobile ions and

$$
C^{\prime}=\left(1 / C_{H}+1 / C_{d}\right)^{-1}
$$

If we neglect the image interaction $\frac{48}{}$, the electrostatic free energy $F_{e}$ appropriate at fixed $\sigma_{m}$ is the space integral of $D E / 8 \pi$ in the whole region $(-\ell<z<H)$. Then,

$$
\begin{aligned}
F_{e} & =\int_{d_{0}}^{H-d_{H}} d z \frac{\varepsilon_{0} E^{2}}{8 \pi}+\frac{\left(\sigma_{A}+\sigma_{m}\right)^{2}}{2 C_{0}}+\frac{\sigma_{m}^{2}}{2 C^{\prime}} \\
& =\frac{1}{2} \int_{0}^{H} d z \rho \Phi+\frac{1}{2} \sigma_{\mathrm{A}} \Phi(0)+\frac{1}{2} \sigma_{m} V .
\end{aligned}
$$

We rewrite the integral in the first line as $\left[\Phi\left(d_{0}\right) D\left(d_{0}\right)-\right.$ $\left.\Phi\left(H-d_{0}\right) D\left(H-d_{0}\right)\right] / 8 \pi+\int d z \Phi \rho / 2$ and use Eq. (20). It then follows the second line, where the integration region $d_{0}<z<H-d_{H}$ has been changed to $0<z<H$ and $\sigma_{\mathrm{A}}$ is related to $\rho$ by Eq.(13). Note that the integrals of $\rho$ in the Stern layers are assumed to be negligible.

We also consider small changes $\rho \rightarrow \rho+\delta \rho$ and $\sigma_{m} \rightarrow$ $\sigma_{m}+\delta \sigma_{m}$. The incremental change in $F_{e}$ is written as

$$
\delta F_{e}=\int_{0}^{H} d z \Phi \delta \rho+\Phi(0) \delta \sigma_{A}+V \delta \sigma_{m},
$$

where $\delta \sigma_{A}=-\int_{0}^{H} d z \delta \rho(z)$ from Eq.(13) and the last term vanishes at fixed $\sigma_{m}$. On the other hand, at fixed $V$, the appropriate free energy is $F_{e}-\sigma_{m} V$, whose differential form is given by the first two terms in Eq.(24). Here, we neglect the ion-ion correlation due to the fluctuations in the $x y$ plane and the image interaction between the ions and the image charges in the solid regions.

In our analysis, we use the following capacitance values. (i) For our dielectric film, we assume $\varepsilon_{d}=4$ and $\ell=1.05 \mathrm{~nm}$ to obtain $C_{d}=0.0335 \mathrm{~F} / \mathrm{m}^{2}$ from Eq.(17). On the other hand, in analysis of electrowetting, Klarman and Andelman $\underline{49}$ assumed a much smaller value, $C_{d}=4.4 \times 10^{-6} \mathrm{~F} / \mathrm{m}^{2}$, for a film with $\varepsilon_{d}=2.67$ and $\ell=5 \mu \mathrm{m}$. In their case, the relation $V \cong \sigma_{m} / C_{d}$ held nicely for not very small $V$. (ii) Supposing a silica-water interface $e^{7 / 17}\left[19\right.$, we set $C_{0}=2.9 \mathrm{~F} / \mathrm{m}^{2}$. (iii) The metalwater capacitance ${ }^{40 / 44}$ has been observed in the range $0.2-0.5 \mathrm{~F} / \mathrm{m}^{2}$, so we set $C_{H}=0.3 \mathrm{~F} / \mathrm{m}^{2}$. Then, $1 / C_{H}=$ $0.11 / C_{d}$ in our case. Via microscopic simulations $41 / 44 \mid 45$, the surface capacitance for a metal-water interface has been calculated in a range of $0.05-0.1 \mathrm{~F} / \mathrm{m}^{2}$ with a layer thickness about $5 \AA$ (where water molecules are depleted).

\section{Free energy functional}

We set up the Helmholtz free energy functional $F$ for a cell with $d_{0} \ll H$ and $d_{H} \ll H$ in the mean field theory. At fixed $\sigma_{m}$, it consists of three parts as

$$
F=F_{e}+F_{b}+F_{s} .
$$

At fixed $V$, we should replace $F_{e}$ by $F_{e}-\sigma_{m} V$. Here, $F_{b}$ is the contribution from the solute particles and $F_{s}$ is that for the surface ionization:

$$
\begin{aligned}
F_{b} / T= & \int_{0}^{H} d z\left[\sum_{i} n_{i}\left[\ln \left(n_{i} \lambda_{i}^{3}\right)-1\right]+\Delta_{\mathrm{b}} n_{\mathrm{M}}\right. \\
& \left.+\Delta_{\mathrm{w}}\left(n_{\mathrm{OH}}-n_{\mathrm{M}}\right)\right], \\
F_{s} / T \Gamma_{0}= & \alpha \ln \alpha+(1-\alpha) \ln (1-\alpha)+\Delta_{\sigma} \alpha,
\end{aligned}
$$

where $\lambda_{i}\left(\propto T^{1 / 2}\right)$ is the thermal de Broglie length of the particle species $i$. The $T \Delta_{\mathrm{b}}, T \Delta_{\mathrm{w}}$, and $T \Delta_{s}$ are the dissociation free energies for the chemical reactions in Eqs.(1), (2), and (9), respectively, which arise from the microscopic interactions. The above form of $F_{s}$ has long been used in the literature $30|32| 35|36| 47$, where the first two terms are entropic contributions.

We define the chemical potentials of the solute particles by $\mu_{i}=\delta F / \delta n_{i}$ at fixed $\alpha$. Then, Eqs.(25)-(27) give

$$
\begin{aligned}
& \mu_{\mathrm{MOH}} / T=\ln \left[n_{\mathrm{MOH}} \lambda_{\mathrm{MOH}}^{3}\right], \\
& \mu_{\mathrm{M}} / T=\ln \left[n_{\mathrm{M}} \lambda_{\mathrm{M}}^{3}\right]+\Delta_{\mathrm{b}}-\Delta_{\mathrm{w}}+U, \\
& \mu_{\mathrm{OH}} / T=\ln \left[n_{\mathrm{OH}} \lambda_{\mathrm{OH}}^{3}\right]+\Delta_{\mathrm{w}}-U, \\
& \mu_{\mathrm{H}} / T=\ln \left[n_{\mathrm{H}} \lambda_{\mathrm{H}}^{3}\right]+U,
\end{aligned}
$$

where $U(z)=e \Phi(z) / T$ is the normalized potential.

In equilibrium, we require the charge neutrality condition (12). Here, we can either assume Eq.(14) with a constant $\bar{n}$ in a closed cell or attach a reservoir to the cell with a common $\mu_{\mathrm{MOH}}$ (see Appendix $\left.\mathrm{B}\right)^{15}$. In these cases, we should minimize the grand potential,

$$
\begin{aligned}
\Omega & =F-h_{0}\left(N_{\mathrm{M}}+N_{\mathrm{MOH}}\right) \\
& -h_{1}\left(N_{\mathrm{H}}-N_{\mathrm{OH}}+N_{\mathrm{M}}-\Gamma_{0} \alpha\right),
\end{aligned}
$$

where $h_{0}$ and $h_{1}$ are homogeneous constants. Requiring $\delta \Omega / \delta n_{i}=0$, we obtain the chemical equilibrium conditions for the mobile particles:

$$
\begin{aligned}
& \mu_{\mathrm{MOH}}=\mu_{\mathrm{M}}+\mu_{\mathrm{OH}}=h_{0}, \\
& \mu_{\mathrm{H}}=-\mu_{\mathrm{OH}}=h_{1} .
\end{aligned}
$$

With these relations, $\Omega$ can be expressed as

$$
\Omega=F-\sum_{i \neq \mathrm{H}} \mu_{i} N_{i}-\mu_{\mathrm{H}}\left(N_{\mathrm{H}}-\Gamma_{0} \alpha\right) .
$$

See Appendix D for more detailed expressions of $\Omega$.

Assuming the equilibrium conditions in Eqs.(33) and (34) for $n_{i}(z)$, we may treat $\Omega=\Omega(\alpha)$ as a function of 
$\alpha$. Then, its minimization with respect to $\alpha$ gives the equilibrium $\alpha$. From Eq.(27) we obtain its derivative,

$$
\frac{1}{T \Gamma_{0}} \frac{d \Omega}{d \alpha}=\ln \left(\frac{\alpha}{1-\alpha}\right)+\Delta_{s}+\ln \left[n_{\mathrm{H}}(0) \lambda_{\mathrm{H}}^{3}\right]
$$

Using Eqs.(33) and (34) and setting $d \Omega / d \alpha=0$, we derive the chemical equilibrium conditions (4), (5), and (11) with the dissociation constants,

$$
\begin{aligned}
& K_{\mathrm{b}}=\left(\lambda_{\mathrm{MOH}} / \lambda_{\mathrm{H}} \lambda_{\mathrm{OH}}\right)^{3} \exp \left(-\Delta_{\mathrm{b}}\right), \\
& K_{\mathrm{w}}=\left(\lambda_{\mathrm{H}} \lambda_{\mathrm{OH}}\right)^{-3} \exp \left(-\Delta_{\mathrm{w}}\right), \\
& K_{s}=\lambda_{\mathrm{H}}^{-3} \exp \left(-\Delta_{s}\right) .
\end{aligned}
$$

With Eq.(39) the right hand side of Eq.(36) becomes $\ln \left[\alpha n_{\mathrm{H}}(0) /(1-\alpha) K_{s}\right]$, leading to Eq.(11) in equilibrium.

If we use $K_{b}$ for $\mathrm{NaOH}$ and $K_{s}$ for silica, Eqs.(37)-(39) give $\Delta_{\mathrm{b}}=8.4, \Delta_{\mathrm{w}}=51$, and $\Delta_{s}=24$. These large sizes of $\Delta_{\mathrm{w}}$ and $\Delta_{s}$ indicate that the autoionization in water and the dissociation on a silica oxide surface are rare activation processes. On the other hand, $\Delta_{\mathrm{b}}$ is relatively small such that $\mathrm{NaOH}$ mostly dissociates in water.

We previously presented free energies with variable charges in mixture solvents for colloidal particles 30 , rods 34 , and polyelectrolytes ${ }^{35}$ (without applied field). In such systems, if $\Delta_{s}$ depends on the composition, ionization and wetting transitions are coupled.

\section{Space-dependence of ion densities}

From Eqs.(28)-(31), $n_{\mathrm{MOH}}$ is a homogeneous constant. The ion densities can be expressed in terms of $U(z)$ as

$$
\begin{aligned}
& n_{\mathrm{OH}}(z)=n_{\mathrm{OH}}^{0} e^{U(z)}, \quad n_{\mathrm{H}}(z)=n_{\mathrm{H}}^{0} e^{-U(z)} \\
& n_{\mathrm{M}}(z)=n_{\mathrm{M}}^{0} e^{-U(z)}
\end{aligned}
$$

where $n_{i}^{0}$ are constants with $n_{\mathrm{M}}^{0}+n_{\mathrm{H}}^{0}=n_{\mathrm{OH}}^{0}$. See Appendix $\mathrm{B}$ for more details on these expressions. From Eq.(18) we obtain the PB equation,

$$
d^{2} U / d z^{2}=\kappa^{2} \sinh (U)
$$

We define the Debye wave number $\kappa$ by

$$
\kappa=\left(8 \pi \ell_{B} n_{\mathrm{OH}}^{0}\right)^{1 / 2}=10^{-3}\left(n_{\mathrm{OH}}^{0} / K_{\mathrm{w}}^{1 / 2}\right)^{1 / 2} / \mathrm{nm} .
$$

where $\ell_{B}=e^{2} / \varepsilon_{0} T=7.0 \AA$ is the Bjerrum length. The inverse $\kappa^{-1}$ is the Debye length, which is long here, but it can be shortened if we add a salt such as $\mathrm{KCl}$. In such cases, $n_{\mathrm{OH}}^{0}$ in Eq.(42) should be replaced by the total anion density $n_{\mathrm{b}}^{0}$ in the bulk.

For $\kappa H \gg 1, n_{i}^{0}$ are the bulk ion densities far from the walls as in Eqs.(6)-(8), where the charge neutrality condition holds. However, even for not large $\kappa H$, we can use Eq.(40) with well-defined $n_{i}^{0}$. Under Eq.(14) in a closed cell, $n_{\mathrm{M}}^{0}$ is determined by $n_{\mathrm{M}}^{0} \int_{0}^{H} d z e^{-U} / H+$ $n_{\mathrm{MOH}}=\bar{n}$ (see Eq.(58)). If we attach a reservoir to the cell with a common $n_{\mathrm{MOH}}, n_{i}^{0}$ in Eq.(40) are simply equal to the ion densities in the reservoir (see Appendix B).

The protons are assumed to penetrate into the Stern layers. From Eq.(19) the ratio of its values at $z=0$ and $d_{0}$ is given by the Boltzmann factor $\exp \left(-e V_{0}^{\mathrm{S}} / T\right)$, so

$$
n_{\mathrm{H}}(0) / n_{\mathrm{H}}\left(d_{0}\right)=\exp \left[-e\left(\sigma_{\mathrm{A}}+\sigma_{m}\right) / C_{0} T\right] .
$$

This relation was proposed by Behrens et al! $17-19$, but it should be checked with microscopic simulations.

\section{E. Osmotic and disjoining pressures}

In our system, the osmotic pressure $\Pi$ is defined in the bulk region $d_{0}<z<H-d_{H}$ and is given by

$$
\Pi=T \sum_{i} n_{i}(z)-\frac{\varepsilon_{0}}{8 \pi} E(z)^{2},
$$

where the first term is the partial pressure of the solute particles and the second term is the $z z$ component of the

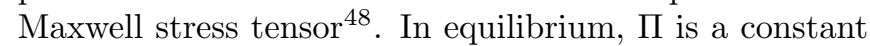
independent of $z$. In fact, $d \Pi / d z=0$ from Eqs.(18) and (40). Here, we assume $H>d_{0}+d_{H} \sim 1 \mathrm{~nm}$.

Let us treat the equilibrium (minimum) value of $\Omega$ under Eqs.(11), (33), and (34) as a function of the cell width $H$ in the fixed charge or the fixed potential condition. In Appendix C, we will derive the following relation,

$$
\Pi=-\frac{\partial}{\partial H} \Omega \text {. }
$$

A similar formula holds for the force between two colloidal particles with $H$ being their separation distance ${ }^{51}$. As will be shown in Appendix C, Eq.(45) holds when $n_{\mathrm{MOH}}$ or $h_{0}=\mu_{\mathrm{MOH}}$ in Eq.(33) is fixed in the derivative. This is equivalent to fixing $n_{\mathrm{M}} n_{\mathrm{OH}}$ from Eq.(4). It can be realized if we attach a reservoir to the cell. On the other hand, if the cell is closed and $\bar{n}$ in Eq.(14) is fixed, Eq.(C5) in Appendix C leads to $\Pi=-\partial F / \partial H$.

We suppose a reservoir without applied field. The $n_{\mathrm{MOH}}$ in the reservoir is the same as that in the cell. Then, the disjoining pressure is the difference $\Pi_{d}=$ $\Pi-\Pi_{r}^{0}$, where $\Pi_{r}^{0}$ is the osmotic pressure in the reservoir (see Appendix B). Using $n_{\mathrm{OH}}^{0}$ in Eq.(40), we find ${ }^{1 / 4 \mid 22}$

$$
\Pi_{d}=T n_{\mathrm{OH}}^{0}\left[2 \cosh (U)-2-\kappa^{-2}|d U / d z|^{2}\right] .
$$

Thus, $\Pi_{d}=-\partial\left(\Omega+H \Pi_{r}^{0}\right) / \partial H$ at fixed $n_{\mathrm{MOH}}$. In the presence of other kinds of monovalent ions, $n_{\mathrm{OH}}^{0}$ in Eq.(46) should be replaced by the total anion (cation) density $n_{\mathrm{b}}^{0}$ in the reservoir, as stated below Eq.(42).

\section{IONIZATION ON SILICA SURFACE AT LARGE WALL SEPARATION}

We consider equilibrium in a thick cell with $H \gg \kappa^{-1}$, $d_{0}$, and $d_{H}$. In the middle region $z \gg \kappa^{-1}$ and $H-$ $z \gg \kappa^{-1}$, the electrolyte is homogeneous with bulk ion densities $n_{i}^{0}$. 


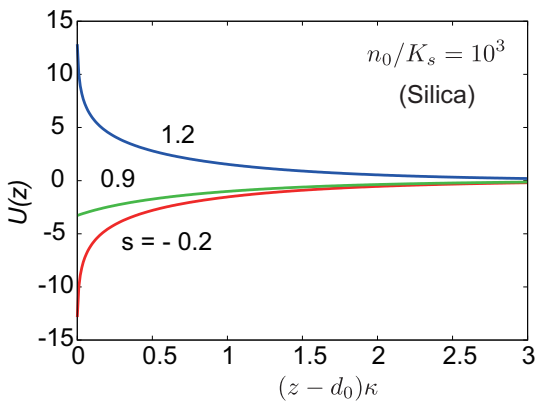

FIG. 3: Normalized electric potential $U(z)$ above the Stern layer $\left(z>d_{0}\right)$ near the bottom wall at $A_{1}=1550\left(n_{0} / K_{s}=\right.$ $10^{3}$ for silica), where $\alpha$ depends on $s=\sigma_{m} / e \Gamma_{0}$. For three curves the initial slope at $z=d_{0}$ is $2 A_{1} u$ with $u=\alpha-s$, where $(s, \alpha)=\left(-0.20,2.2 \times 10^{-5}\right),(0.90,0.902)$, and $(1.2,1.0)$ from below. Thus, $u=0.20,0.002$, and -0.2 , respectively. For the middle curve $(s=0.90), 0<u \ll 1$ due to self-regulation. For the other curves the initial slope is steep.

\section{A. Basic solution for large $H$}

For $H \gg \kappa^{-1}$, we impose the semi-infinite boundary condition: $U(z) \rightarrow 0$ far from the walls. Near the bottom and the top, the profiles of the diffuse layers are given by

$$
\begin{array}{rll}
U(z) & =2 \ln \left[\frac{1-\eta e^{-\kappa\left(z-d_{0}\right)}}{1+\eta e^{-\kappa\left(z-d_{0}\right)}}\right] \quad \text { (bottom) } \\
& =2 \ln \left[\frac{1-\zeta e^{\kappa\left(z-H+d_{H}\right)}}{1+\zeta e^{\kappa\left(z-H+d_{H}\right)}}\right] \quad \text { (top). }
\end{array}
$$

From Eq.(20) the coefficients $\eta$ and $\zeta$ are determined by

$$
\begin{aligned}
& 2 \eta /\left(1-\eta^{2}\right)=-\left(2 \pi \ell_{B} / e \kappa\right)\left(\sigma_{\mathrm{A}}+\sigma_{m}\right), \\
& 2 \zeta /\left(1-\zeta^{2}\right)=\left(2 \pi \ell_{B} / e \kappa\right) \sigma_{m} .
\end{aligned}
$$

We define dimensionless parameters $A_{1}, A_{2}, u$, and $s$ by

$$
\begin{aligned}
& A_{1}=2 \pi \ell_{B} \Gamma_{0} / \kappa=\Gamma_{0}\left(\pi \ell_{B} / 2 n_{\mathrm{OH}}^{0}\right)^{1 / 2}, \\
& A_{2}=e^{2} \Gamma_{0} / C_{0} T \\
& u=-\left(\sigma_{\mathrm{A}}+\sigma_{m}\right) / e \Gamma_{0}=\alpha-s, \\
& s=\sigma_{m} / e \Gamma_{0} .
\end{aligned}
$$

Then, the right hand side of Eq.(48) is $A_{1} u$ and that of Eq.(49) is $A_{1} s$. From Eq.(20), $d U / d z$ is $2 A_{1} u$ at $z=d_{0}$ and is $-2 A_{1} s$ at $z=H-d_{H}$ as the boundary conditions.

In terms of these parameters the total potential difference in Eq.(21) is rewritten as

$$
\frac{e}{T} V=-g\left(A_{1} u\right)-A_{2} u+g\left(A_{1} s\right)+\frac{e \sigma_{m}}{T C^{\prime}} .
$$

where $g(x)=2 \ln \left[\sqrt{1+x^{2}}+x\right]$. Then, $g(-x)=-g(x)$. In Eq. (54) the first term $\left(=U\left(d_{0}\right)\right)$ arises from the lower diffuse layer, the second from the lower Stern layer, the third $\left(=-U\left(H-d_{H}\right)\right)$ from the upper diffuse layer, and the last term is due to the dielectric film and the upper Stern layer with Eq.(22). Here, the Debye-Hückel theory is valid only for $|u| \ll A_{1}^{-1}$ at the bottom and for $|s| \ll A_{1}^{-1}$ at the top. For silica, we have $A_{1}=$ $4.9 \times 10^{4}\left(K_{s} / n_{\mathrm{OH}}^{0}\right)^{1 / 2}$ and $A_{2}=17$. Thus, $A_{1} \gg 1$ for realistic $n_{\mathrm{OH}}^{0}$ because of large $\Gamma_{0}$ and small $\kappa$.

Ninham and Parsegian $\frac{15}{15}$ set $\mathrm{pK}=4.8, \Gamma_{0}=1$ and $0.25 / \mathrm{nm}^{2}$, and $\kappa=1.29 / \mathrm{nm}$ with a salt added. As a result, $A_{1}$ in Eq.(50) was of order 1 and $\kappa^{-1}$ did not exceed the Gouy-Chapman length (see Eq.(56) below).

\section{B. Profiles in the nonlinear $\mathrm{PB}$ regime}

There appear three cases with increasing $s$ : (i) $s<0$ and $u>0$ with $d U / d z>0$, (ii) $s>0$ and $u>0$ with $U(z)$ lower near the walls than in the middle, and (iii) $s>0$ and $u<0$ with $d U / d z<0$. We are interested in the nonlinear behavior of $\alpha$ in case (ii), since $\alpha \rightarrow 0$ in case (i) and $\alpha \rightarrow 1$ in case (iii).

In Fig.3, we display $U(z)$ near the bottom for $A_{1}=$ $1.55 \times 10^{3}$ (at $n_{0} / K_{s}=10^{3}$ for silica), where $\alpha$ is determined for given $n_{0}$ and $s$. Then, $(s, \alpha)=(-0.20,2.2 \times$ $\left.10^{-5}\right),(0.90,0.902)$, and $(1.2,1.0)$. In the case of $s=0.9$, $u$ is small $(=0.002)$ or $\left(\sigma_{\mathrm{A}}+\sigma_{m}\right) / \sigma_{m} \cong-0.002$, but $A_{1} u=3.1$ in the nonlinear $\mathrm{PB}$ regime. We shall see that this charge cancellation is a universal effect.

Let us consider the profile of $U$ and $n_{\mathrm{H}}$ at the bottom in the nonlinear PB regime $A_{1} u \gg 1$ with $u>0$. The coefficient $\eta$ in Eq.(48) is close to 1 as $\eta \cong 1+1 / A_{1} u$, so

$$
\begin{aligned}
& U(z) \cong 2 \ln \left[\left(\kappa\left(z-d_{0}+\ell_{\mathrm{GC}}\right) / 2\right],\right. \\
& n_{\mathrm{H}}(z) / n_{\mathrm{H}}^{0} \cong 4 \kappa^{-2}\left(z-d_{0}+\ell_{\mathrm{GC}}\right)^{-2} .
\end{aligned}
$$

Here, $\ell_{\mathrm{GC}}$ is the Gouy-Chapman length $\frac{28}{28}$ at the bottom,

$$
\ell_{\mathrm{GC}}=\left(\kappa A_{1}|u|\right)^{-1}=e /\left(2 \pi \ell_{B}\left|\sigma_{\mathrm{A}}+\sigma_{m}\right|\right),
$$

where we assume $\ell_{\mathrm{GC}} \ll \kappa^{-1}$. The profiles change on the scale of $\ell_{\mathrm{GC}}$. In this case, $n_{\mathrm{H}}\left(d_{0}\right)$ is larger than $n_{\mathrm{H}}^{0}$ as

$$
n_{\mathrm{H}}\left(d_{0}\right) / n_{\mathrm{H}}^{0} \cong\left(2 A_{i} u\right)^{2} .
$$

The ratio $n_{\mathrm{M}}\left(d_{0}\right) / n_{\mathrm{M}}^{0}$ is also given by the right hand side of Eq. (57), which is important for $n_{\mathrm{M}}^{0} \cong n_{\mathrm{OH}}^{0} \gg n_{\mathrm{H}}^{0}$.

We also calculate the integral $N_{\mathrm{H}}=\int_{o}^{H} d z n_{\mathrm{H}}(z)$ using Eqs.(40) and (47). For $\kappa H \gg 1$, we thus obtain

$$
\begin{aligned}
& N_{\mathrm{H}} / H n_{\mathrm{H}}^{0}-1=2 A_{1} \alpha / \kappa H \\
& +2\left[\left(A_{1}^{2} u^{2}+1\right)^{1 / 2}+\left(A_{1}^{2} s^{2}+1\right)^{1 / 2}-2\right] / \kappa H .
\end{aligned}
$$

The right hand side is the correction $\left(\propto H^{-1}\right)$. It can also be equated with $N_{\mathrm{M}} / H n_{\mathrm{M}}^{0}-1$ for $\mathrm{M}^{+}$and $\left(N_{\mathrm{OH}}+\right.$ $\left.\Gamma_{0} \alpha\right) / H n_{\mathrm{OH}}^{0}-1$ for $\mathrm{OH}^{-}$, where $N_{\mathrm{M}} / H=\bar{n}-n_{\mathrm{MOH}}$ from Eq.(14) and $n_{\mathrm{M}}^{0}=n_{0}-n_{\mathrm{MOH}}$ from Eq.(7). It follows $n_{\mathrm{H}}^{0} \leq N_{\mathrm{H}} / H$ for any $u$ and $s$. In particular, if $u \gg A_{1}^{-1}$ and $s \gg A_{1}^{-1}$, the right hand side is $4 A_{1} \alpha / \kappa H$ and is negligible only for $H \gg A_{1} \alpha \kappa^{-1}$. Thus, for $\kappa H<A_{1} \alpha$, the ion densities $N_{i} / H$ and $n_{i}^{0}$ are largely different. 


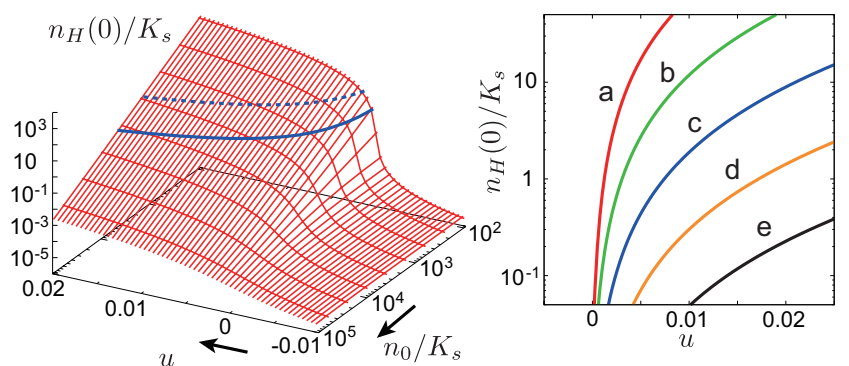

FIG. 4: Left: Ratio $n_{\mathrm{H}}(0) / K_{s}$ from Eq.(59) in the $u-n_{0} / K_{s}$ plane with $u$ in Eq. (52), which is $(1-\alpha) / \alpha$ in equilibrium. On the surface, it is 1 on the lower bold line and is 10 on the upper dotted line, where $\alpha$ is 0.5 and 0.09 , respectively . Right: $n_{\mathrm{H}}(0) / K_{s}$ vs $u$ with $n_{0} / K_{s}$ being (a) $2.5 \times 10^{2}$, (b) $6.3 \times 10^{2}$, (c) $1.6 \times 10^{3}$ (right), (d) $4.0 \times 10^{3}$, and (e) $1.0 \times 10^{4}$ from above, where increase is steep for $n_{0} / K_{s}<10^{-4}$.
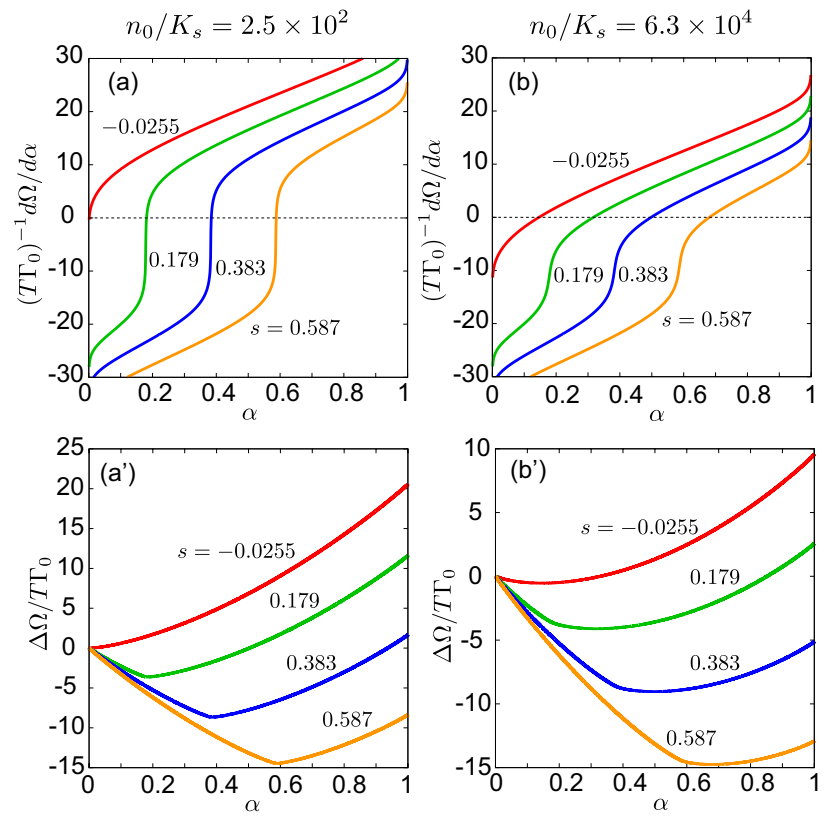

FIG. 5: Left: $d \Omega(\alpha) / d \alpha$ and $\Delta \Omega=\Omega(\alpha)-\Omega(0)$ divided by $T \Gamma_{0}$ as functions of $\alpha$ for $s=\sigma_{m} / e \Gamma_{0}=-0.0255,0.179,0.383$, and 0.587 at $n_{0} / K_{s}=2.5 \times 10^{2}$. Minimization of $\Omega(\alpha)$ gives equilibrium $\alpha$. Right: those at $n_{0} / K_{s}=6.3 \times 10^{4}$.

\section{Numerical results on $\alpha$}

Use of Eqs.(40) and (43) gives the ratio of the surface proton density $n_{\mathrm{H}}(0)$ to the bulk one $n_{\mathrm{H}}^{0}$ as

$$
\begin{aligned}
n_{\mathrm{H}}(0) / n_{\mathrm{H}}^{0} & =\exp \left[g\left(A_{1} u\right)+A_{2} u\right] \\
& =\left[\left(1+A_{1}^{2} u^{2}\right)^{1 / 2}+A_{1} u\right]^{2} e^{A_{2} u},
\end{aligned}
$$

which depends on $u$ and $n_{0}$. The right hand side increases with increasing $u$ being equal to 1 for $u=0$. In Eq,(52), $-u$ denotes the normalized, effective surface charge density at $z=0$. Thus, for $u>0(u<0)$, the protons are more enriched (decreased) near the surface than in the bulk. From $A_{1} \gg 1, n_{\mathrm{H}}(0) / n_{\mathrm{H}}^{0}$ can be of order only in
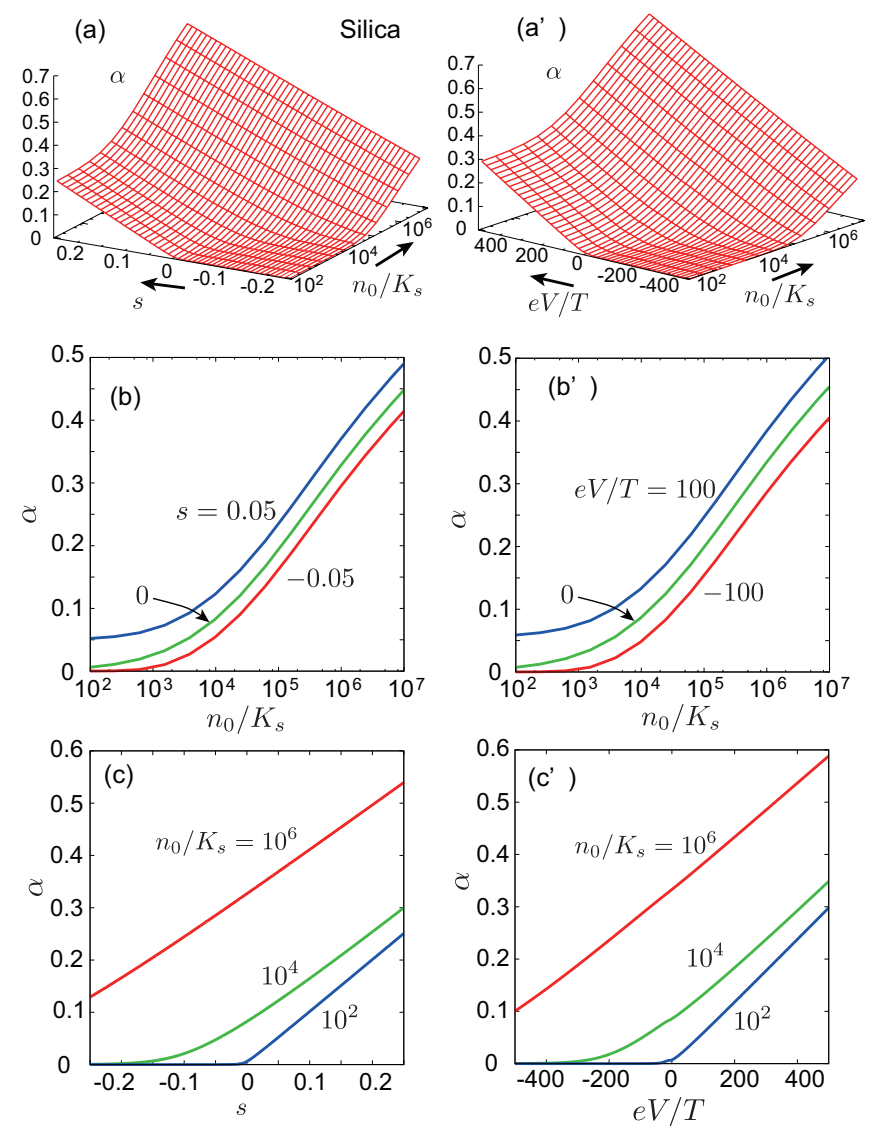

FIG. 6: Equilibrium $\alpha$ for silica in charge-controlled case (left) and in potential-controlled case (right). Top: $\alpha$ in the $s$ $n_{0} / K_{s}$ plane in (a) and in the $\mathrm{eV} / T-n_{0} / K_{s}$ plane in (a'), where $s=\sigma_{m} / e \Gamma_{0}$. Middle: $\alpha$ vs $n_{0} / K_{s}$ at $s=0$ and \pm 0.05 in (b) and $e V / T=0$ and \pm 100 in (b'), where $n_{0} / K_{s}=10^{\mathrm{pH}-6.7}$ in terms of the bulk pH. Bottom: $\alpha$ vs $s$ in (c) and $\alpha$ vs $e V / T$ in (c') for $n_{0} / K_{s}=10^{2}, 10^{4}$, and $10^{6}$, which are nearly linear.

the narrow range $|u|<A_{1}^{-1}$. Outside this range, it grows or decays rapidly depending on the sign of $u$ as

$$
\begin{aligned}
n_{\mathrm{H}}(0) / n_{\mathrm{H}}^{0} \cong\left(2 A_{1} u\right)^{2} e^{A_{2} u} \gg 1 & \left(u \gg A_{1}^{-1}\right) \\
\cong\left(2 A_{1}|u|\right)^{-2} e^{-A_{2}|u|} \ll 1 & \left(-u \gg A_{1}^{-1}\right) .
\end{aligned}
$$

These changes occur in the region $z<\ell_{\mathrm{GC}}$ (see Eq.(57)).

In the surface chemical equilibrium (11), relevant is the ratio $n_{\mathrm{H}}(0) / K_{s}$, which is close to $\left(K_{\mathrm{w}} / K_{s} n_{\mathrm{OH}}^{0}\right)\left(n_{\mathrm{H}}(0) / n_{\mathrm{H}}^{0}\right)$ and is calculated from Eq. $(59)$. In the left panel of Fig.4, it is plotted in the $u-n_{0} / K_{s}$ plane. From Eq.(60), it grows abruptly from small values $(\ll 1)$ to large values $(\gg 1)$ with increasing $u$ above 0 . In the right panel, we show curves of $n_{\mathrm{H}}(0) / K_{s}$ vs $u$ for several $n_{0} / K_{s}$, for which $A_{1}$ is indeed large $\left(2.5 \times 10^{3}\right.$ for curve (a) and $3.9 \times 10^{2}$ for curve (e)). For each point on the surface or the curves in Fig. 4 we can find the corresponding equilibrium by setting $\alpha=\left[1+n_{\mathrm{H}}(0) / K_{s}\right]^{-1}$ and $\sigma_{m}=e \Gamma_{0}(\alpha-u)$.

Substitution of Eq.(59) into Eq.(36) gives $d \Omega(\alpha) / d \alpha$ as a function of $\alpha$ for given $\sigma_{m}$ and $n_{0}$. In Fig.5, we plot it 

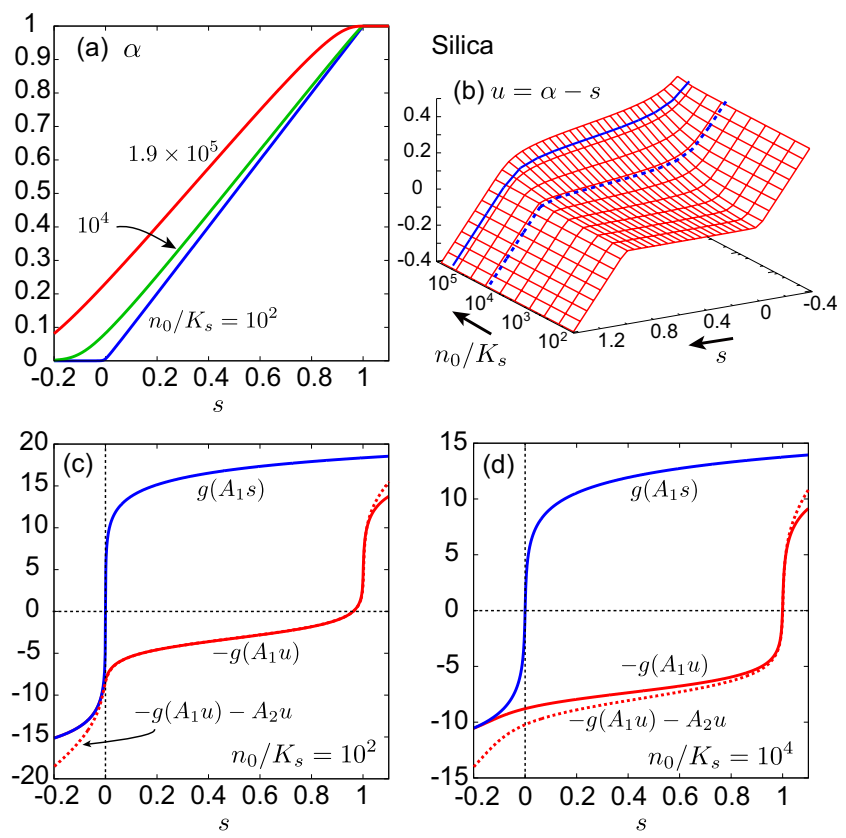

FIG. 7: (a) $\alpha$ vs $s=\sigma_{m} / e \Gamma_{0}$ for $n_{0} / K_{s}=10^{2}, 10^{4}$, and $1.9 \times$ $10^{5}$ in range $[-0.2,1.1]$ for silica, where $\alpha \cong s$. (b) $u=\alpha-s$ in the $s-n_{0} / K_{s}$ plane, which is very small for $0<s<1$ and $n_{0} \ll n_{c}$. The lines (blue) on the surface indicate $n_{0} / n_{c}=0.1$ and 1. (c) Terms in $e V / T$ in Eq.(54) for $n_{0} / K_{s}=10^{2}: g\left(A_{1} s\right)$ from the upper diffuse layer, $-g\left(A_{1} u\right)$ from the lower diffuse layer, and $-g\left(A_{1} u\right)-A_{2} u$ including the lower Stern layer contribution. (d) Those for $n_{0} / K_{s}=10^{4}$. In (c) and (d), the $\mathrm{PB}$ equation can be linearized only for $\left|g\left(A_{1} u\right)\right|<1$ at the bottom and $\left|g\left(A_{1} s\right)\right|<1$ at the top.

and its integral $\Delta \Omega=\int_{0}^{\alpha} d \alpha(d \Omega / d \alpha)=\Omega(\alpha)-\Omega(0)$ as functions of $\alpha$ for four $s=\sigma_{m} / e \Gamma_{0}$. See Appendix D for the explicit expression of $\Delta \Omega$. Minimization of $\Delta \Omega(\alpha)$ yields equilibrium $\alpha$ satisfying Eq.(11). For $n_{0} / K_{s}=$ $2.5 \times 10^{2}$ (left), $d \Omega(\alpha) / d \alpha$ grows abruptly and $\Delta \Omega(\alpha)$ has a cusp-like minimum around $\alpha \cong s=\sigma_{m} / e \Gamma_{0}$ with $0<u \ll 1$ (see Fig.7b). For a larger $n_{0} / K_{s}=6.3 \times$ $10^{4}$ (right), $d \Omega(\alpha) / d \alpha$ grows gradually and $\Delta \Omega(\alpha)$ has a broad minimum.

In Fig.6, we plot the equilibrium $\alpha$ as a function of $\sigma_{m}$ and $n_{0}$ (left) and as a function of $V$ and $n_{0}$ (right), where $\sigma_{m}$ and $V$ are related by Eq.(54). Salient results are as follows. (i) We can see close resemblance between the left and right panels, which suggests an approximate linear relation $V \propto \sigma_{m}$ (see Sec.IIID). In these panels, $\alpha$ increases with increasing $n_{0}$ in agreement with the experiment ${ }^{24}$ 26] (ii) In the middle, we write $\alpha$ vs $n_{0} / K_{s}$, where we fix $s=\sigma_{m} / e \Gamma_{0}$ at 0 and \pm 0.05 in (b) and $e V / T$ at 0 and \pm 100 in (b'). These curves exhibit different behaviors with a crossover at $n_{0} / K_{s} \sim 10^{4}$. The curve of $\sigma_{m}=0$ exhibits the power-law behavior $\alpha \propto n_{0}^{2 / 3}$ for small $n_{0}$ (see Eq.(63)). (iii) In the bottom, at fixed $n_{0}$, we write $\alpha$ vs $s=\sigma_{m} / e \Gamma_{0}$ in (c) and $\alpha$ vs $e V / T$ in (c'), where $\alpha$ increases linearly with increasing $s$ or $e V / T$. See below for its explanation.
Note that the $\alpha-n_{0}$ relation in the $1 \mathrm{D}$ geometry at $s=0$ can be used for colloidal particles with large radius $(\gg$ $\left.\kappa^{-1}\right) \sqrt{17}\left[19\right.$. At $\sigma_{m}=0$, Behrens and Grier $\frac{19}{19}$ calculated the curves of $\alpha \mathrm{vs} \mathrm{pH}$ in agreement with Fig.6b. At the special point $\sigma_{m}=n_{0}=0$, we find $\alpha=4.7 \times 10^{-4}$ and $\sigma_{\mathrm{A}}=-0.06 \mu \mathrm{C} / \mathrm{cm}^{2}$, where the latter is close to its experimental value $-0.08 \mu \mathrm{C} / \mathrm{cm}^{2}$ for silica colloidal particles in pure water 26 .

\section{Equation for $\alpha$ in nonlinear regime}

In the nonlinear $\mathrm{PB}$ regime with $u=\alpha-s \gg A_{1}^{-1}$, Eqs.(11) and (60) give the following equation of $\alpha$,

$$
\begin{aligned}
\alpha u^{2} e^{A_{2} u} /(1-\alpha) & =K_{s} \kappa^{2} /\left[\left(2 \pi \ell_{B} \Gamma_{0}\right)^{2} n_{\mathrm{H}}^{0}\right] \\
& =\left(n_{\mathrm{OH}}^{0} / n_{c}\right)^{2} .
\end{aligned}
$$

In the second line, we set $\kappa^{2} / n_{\mathrm{H}}^{0}=8 \pi \ell_{B}\left(n_{\mathrm{OH}}^{0}\right)^{2} / K_{\mathrm{w}}$ and we introduce a crossover ion density $n_{c}$ by

$$
n_{c}=\left(2 \pi \ell_{B} K_{\mathrm{w}} / K_{s}\right)^{1 / 2} \Gamma_{0} .
$$

For silica with $\mathrm{NaOH}$, we find $n_{c}=0.97 \times 10^{-2} \mathrm{~mol} / \mathrm{L}=$ $1.9 \times 10^{5} K_{s}$ from Eq.(15). For surfaces with smaller pK (much larger $K_{s}$ ), we may add $\mathrm{HCl}$ to increase $n_{\mathrm{H}}^{0}$ (instead of $\mathrm{NaOH}$ ) at densities much larger than $K_{\mathrm{w}}^{1 / 2}$; then, the right hand side of Eq.(61) becomes $K_{\mathrm{w}} / n_{c}^{2}=$ $K_{s} /\left[2 \pi \ell_{B} \Gamma_{0}^{2}\right]$.

We note that Eq.(61) reproduces the curves of $|s| \ll 1$ in Fig.6b, where we can see $\alpha \ll 1$ for $n_{0} \ll n_{c}$. In particular, for $s=0$ and $n_{0} \ll n_{c}$, Eq.(61) gives

$$
\alpha \cong \alpha_{c}=\left(n_{\mathrm{OH}}^{0} / n_{c}\right)^{2 / 3}=\alpha_{c 0}\left(n_{\mathrm{OH}}^{0} / K_{\mathrm{w}}^{1 / 2}\right)^{2 / 3} .
$$

In pure water, $\alpha_{c}$ tends to the following,

$$
\alpha_{c 0}=\left(K_{\mathrm{w}}^{1 / 2} / n_{c}\right)^{2 / 3}=\left(K_{s} / 2 \pi \ell_{B}\right)^{1 / 3} / \Gamma_{0}^{2 / 3},
$$

For silica, we find $\alpha_{c 0}=4.7 \times 10^{-4}=5.4 / A_{1}$ and $U\left(d_{0}\right)=-7.0$, so we are already in the nonlinear $\mathrm{PB}$ regime at $\sigma_{m}=n_{0}=0$. In terms of the bulk $\mathrm{pH}$, we obtain $\log _{10}\left(\alpha / \alpha_{c 0}\right)=2(\mathrm{pH}-7) / 3$. Note that the relation (59) applies to large colloidal particles in the nonlinear $\mathrm{PB}$ regime. On the other hand, for $n_{0}>n_{c}, u$ increases and the factor $e^{A_{2} u}$ becomes important.

Let us assume $n_{0} \ll n_{c}$ for $n_{c} \gg K_{\mathrm{w}}^{1 / 2}$. In Fig.7a, we confirm the behavior $\alpha \cong s$ in the range $0<s<1$, while we have $\alpha \cong 0$ for $s<0$ and $\alpha \cong 1$ for $s>1$. In (b), this behavior can be seen on a flat part of the surface of $u=\alpha-s$ in the $s-n_{0} / K_{s}$ plane. Here, self-regulated ionization is realized, where the surface charge density $\sigma_{\mathrm{A}}$ due to deplotonation nearly cancels the applied surface charge density $\sigma_{m}$. To be more precise, Eq.(61) yields

$$
u=\alpha-s \cong(1-s)^{1 / 2} s^{-1 / 2} n_{\mathrm{OH}}^{0} / n_{c}
$$

which is much smaller than $s(\cong \alpha)$ for $s \gg \alpha_{c}$ with $\alpha_{c}$ being defined by Eq.(63). If $|s|<\alpha_{c}, \alpha$ becomes of order 

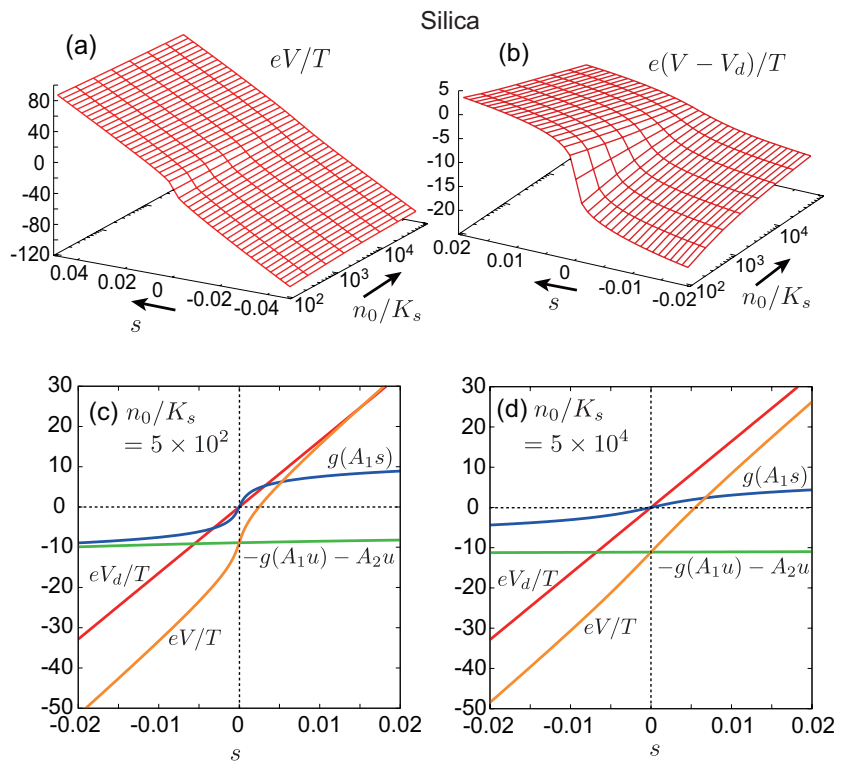

FIG. 8: Potential contributions in Eq.(54) (silica). Top: (a) $e V / T(|s|<0.05)$ and (b) $e\left(V-V_{d}\right) / T(|s|<0.02)$ in the $s-n_{0} / K_{s}$ plane, where $s=\sigma_{m} / e \Gamma_{0}$ and $V_{d}=\sigma_{m} / C^{\prime}$. Bottom: Plotted are $-g\left(A_{1} u\right)-A_{2} u, g\left(A_{1} s\right), V_{d}$, and their sum $V$ as functions of $s$, where $n_{0} / K_{s}$ is (c) $5 \times 10^{2}$ and (d) $5 \times 10^{4}$. See (c) and (d) of Fig. 7 for expanded plots of $-g\left(A_{1} u\right),-g\left(A_{1} u\right)-A_{2} u$, and $g\left(A_{1} s\right)$.

$\alpha_{c}$, which is consistent with Eq.(63). On the other hand, if $n_{c} / K_{\mathrm{w}}^{1 / 2}$ is not large (with smaller $\Gamma_{0} / K_{s}^{1 / 2}$ ), $u$ remains not small for any $n_{0}$.

Finally, we need to require $A_{1} u \gg 1$ self-consistently, which has been assumed in setting up Eq.(61). For $|s| \gg$ $\alpha_{c}$, use of Eqs.(50) and (64) gives

$$
A_{1} u \cong(1-s)^{1 / 2} s^{-1 / 2}\left(n_{\mathrm{OH}}^{0} K_{s} / 4 K_{\mathrm{w}}\right)^{1 / 2} .
$$

If $n_{\mathrm{OH}}^{0} \gtrsim K_{\mathrm{w}} / K_{s}, A_{1} u$ surely exceeds 1 for $s$ not very close to 1 , including the point $s=n_{0}=0$. Thus, it generally follows the self-regulation of surface ionization for $n_{0} \ll n_{c}$ and $0<\sigma_{m}<e \Gamma_{0}$.

\section{E. Potential difference $V$ between electrodes}

In Appendix A, we will explain experimental setups at fixed $V$ and $\sigma_{m}$, so we should compare the results from these two boundary conditions. So far we have found that the right and left panels in Fig.6 look similar.

In Eq.(54) the contributions to $\mathrm{eV} / T$ are written explicitly. In the bottom panels of Fig.7, we examine them in the range $-0.2<s=\sigma_{m} / e \Gamma_{0}<1.1$ as in the upper panels. Here, $g\left(A_{1} s\right)$ is from the upper diffuse layer, $-g\left(A_{1} u\right)$ is from the lower diffuse layer, and $-g\left(A_{1} u\right)-A_{2} u$ includes the lower Stern layer contribution, where $n_{0} / K_{s}$ is $10^{2}$ in (c) and $10^{4}$ in (d). For positive $s$ not close to 0 , the contribution from the lower diffusive layer is smaller than that from the upper one
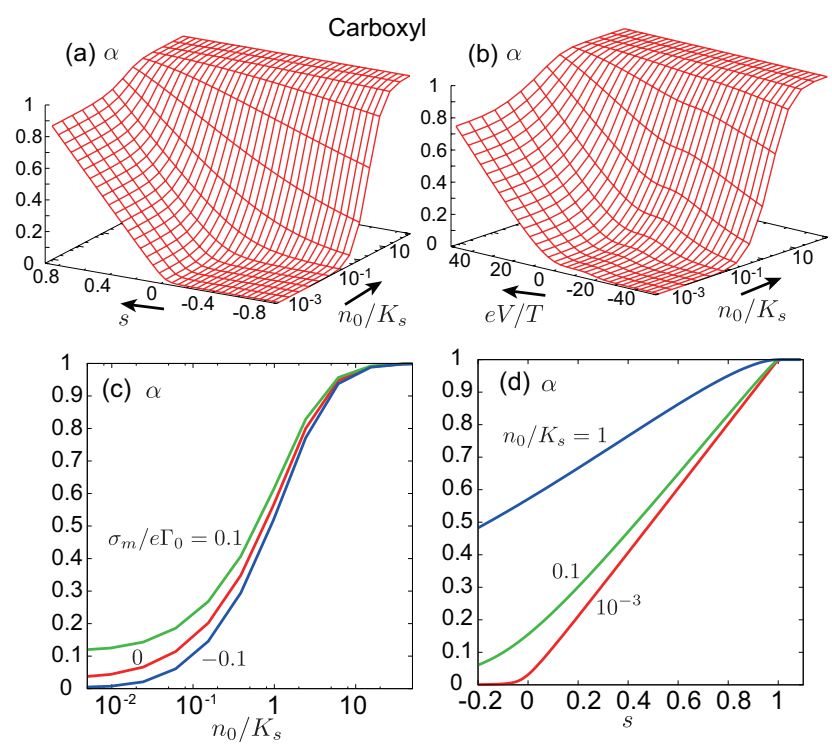

FIG. 9: Ionization on carboxyl-bearing surface. (a) $\alpha$ in the $s-n_{0} / K_{s}$ plane and (b) $\alpha$ in the $e V / T-n_{0} / K_{s}$ plane, where $s=\sigma_{m} / e \Gamma_{0}$. (c) $\alpha$ vs $n_{0} / K_{\sigma}$ at $s=0$ and \pm 0.1 . (d) $\alpha$ vs $s$ for $n_{0} / K_{s}=10^{-3}, 0.1$, and 1 in range $[-0.2,1.1]$.

in magnitude, which is consistent with Fig.7b. Here, for $u \gg A_{1}^{-1}$ and $s \gg A_{1}^{-1}$, we have $U\left(d_{0}\right) \cong-2 \ln \left(2 A_{1} u\right)$ and $U\left(H-d_{H}\right) \cong-2 \ln \left(2 A_{1} s\right)$. We are thus in the nonlinear $\mathrm{PB}$ regime for most $s$. The contribution $-A_{2} u$ from the lower Stern layer is appreciable for $s<0$ and $0<s \ll 1$ or for $\alpha \ll 1$.

In Fig.8, we display (a) $e V / T$ and (b) $e\left(V-V_{d}\right) / T$ in the $s-n_{0} / K_{s}$ plane with $V_{d}=\sigma_{m} / C^{\prime}$, where the surfaces are rather flat for not very small $s$. We also compare $\mathrm{eV} / T, e V_{d} / T, g\left(A_{1} s\right)$, and $-g\left(A_{1} u\right)-A_{2} u$ in the narrow range $|s|<0.02$ for $n_{0} / K_{s}=5 \times 10^{2}$ in (c) and $10^{4}$ in (d). We recognize that $V$ is close to $V_{d}$ except for small $\sigma_{m}$ even for our choice $\ell=1.05 \mathrm{~nm}$. With further increasing $\ell$, the film contribution $\sigma_{m} / C_{d}$ becomes more dominant in $V$, as in electrowetting experiments $\$ 490$.

\section{IONIZATION ON CARBOXYL-BEARING SURFACE AT LARGE WALL SEPARATION}

The carboxyl surface groups undergo the dissociation $\left(\mathrm{COOH} \rightleftarrows \mathrm{COO}^{-}+\mathrm{H}^{+}\right)$at $\mathrm{pK}=4.9$ with a much smaller $\Gamma_{0}$ in water. In their analysis, Behrens et $a^{117-19}$ used two values, $\Gamma_{0}=0.574$ and $0.250 \mathrm{~nm}^{-2}$, for carboxyl-bearing surfaces. These $\mathrm{pK}$ and $\Gamma_{0}$ are very different from those in Eq.(15) for silica. It is worth noting that Aoki et al ${ }^{52}$ fabricated a carboxyl functionalized latex film with coalescence of latex particles on a $\mathrm{Pt}$ electrode. Analysis has also been made on other surfaces such as iron oxide ones ${ }^{14 / 17 / 19}$, which can be positively charged at low $\mathrm{pH}$ due to protonation.

In this section, we examine the equilibrium ionization on a carboxyl-bearing film in the 1D geometry in Fig.1 


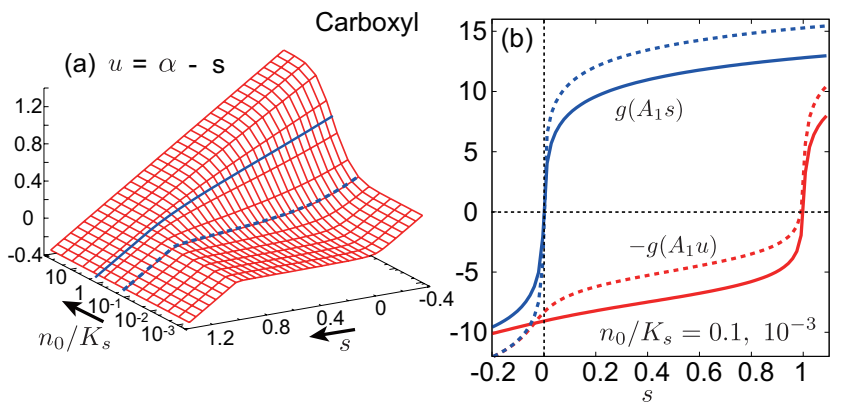

FIG. 10: (a) $u=\alpha-s$ in the $s-n_{0} / K_{s}$ plane for carboxylbearing surface, which is very small for $0<s<1$ and $n_{0}<$ $0.1 n_{c} \sim K_{s}$. The lines (blue) on the surface indicate $n_{0} / n_{c}=$ 0.1 and 1. (b) Terms in $e V / T$ in Eq.(54): $g\left(A_{1} s\right)$ from the upper diffuse layer and $-g\left(A_{1} u\right)$ from the lower diffuse layer. for $n_{0} / K_{s}=0.1$ (bold line) and $10^{-3}$ (dotted line).

with addition of $\mathrm{NaOH}$ in applied field. We set

$$
\begin{aligned}
& K_{s}=10^{-4.9} \mathrm{~mol} / \mathrm{L}=7.5 \times 10^{-6} / \mathrm{nm}^{3}, \\
& \Gamma_{0}=0,25 / \mathrm{nm}^{2} \quad(\text { carboxyl-bearing surface }) .
\end{aligned}
$$

Here, $K_{s}$ is much larger than $K_{\mathrm{w}}^{1 / 2}$ by $10^{2.1}=126$. According to Behrens et al $]^{17] 19}$, the Stern capacitance at carboxyl-bearing surfaces is larger than that for silica oxide surfaces such that its presence itself can be neglected. In fact, for $C_{0}=10 \mathrm{~F} / \mathrm{m}^{2}, A_{2}$ in Eq.(51) is of order 0.1 so $e^{A_{2} u} \cong 1$ in Eq.(59) for $|u| \lesssim 1$. The other parameters are the same as in the silica case in Sec.III.

We use the PB theory in the previous section for large $H$. The parameter $A_{1}$ in Eq.(50) becomes $A_{1}=1.5 \times$ $10^{3}\left(K_{s} / n_{\mathrm{OH}}^{0}\right)^{1 / 2}$. In Eq.(61) we neglect the Stern factor $e^{A_{2} u}$ and assume $u=\alpha-s \gg A_{1}^{-1}$ to obtain

$$
\alpha u^{2} /(1-\alpha) \cong\left(n_{\mathrm{OH}}^{0} / n_{c}\right)^{2},
$$

where $n_{c}=1.9 \times 10^{-5} \mathrm{~mol} / \mathrm{L}=1.5 K_{s}$ from Eq.(67). It is smaller than that for silica by $500^{-1}$. At the special point $n_{0}=\sigma_{m}=0$, our numerical analysis indicates $U(0)=-8.32$ and $\alpha_{c 0}=0.0 a 30=32 / A_{1}$, where the latter nearly coincides with $\alpha_{c 0}$ in Eq.(64). We are in the nonlinear $\mathrm{PB}$ regime at this point.

In Fig.9, we show $\alpha$ in (a) the $s-n_{0} / K_{s}$ plane and in (b) the $e V / T-n_{0} / K_{s}$ plane, as in (a) and (a') of Fig.6. Here, $\log _{10}\left(n_{0} / K_{)}=\mathrm{pH}-9.1\right.$. These look similar as in Fig.6, indicating the linear relation $V \cong \sigma / C^{\prime}$ except for small $\sigma_{m}$. In (c), $\alpha$ vs $n_{0} / K_{s}$ is plotted for $s=0$ and \pm 0.1 , as in Fig.6b. Here, $\alpha$ approaches 1 for $n_{0} \sim 6 K_{s} \sim$ $4 n_{c}$. The curve at $\sigma_{m}=0$ coincides with that calculated by Behrens and Grier ${ }^{19}$. In (d), we display $\alpha$ vs $s$ for $n_{0} / K_{s}=10^{-3}, 0.1$, and 1 in the range $-0.2, s<1.1$, as in Fig.7a. We can again find the linear behavior $\alpha \cong s$ for $0<s<1$ for $n_{0} \ll n_{c}$.

In Fig.10, displayed is (a) $u=\alpha-s$ in the $s-n_{0} / K_{s}$ plane for carboxyl-bearing surface, which is very small for $0<s<1$ and $n_{0}<0.1 n_{c} \sim / K_{s}$, as in Fig.7b. The lines (blue) on the surface indicate $n_{0} / n_{c}=0.1$ and 1 . (b)
Terms in $e V / T$ in Eq.(54) for $n_{0} / K_{s}=10^{2}: g\left(A_{1} s\right)$ from the upper diffuse layer, $-g\left(A_{1} u\right)$ from the lower diffuse layer. for $n_{0} / K_{s}=0.1$ (bold line) and $10^{-3}$ (dotted line).

\section{IONIZATION AND DISJOINING PRESSURE AT SMALL WALL SEPARATION}

The disjoining pressure $\Pi_{d}$ in Eq.(46) is a measurable qunatity 11113. It has been calculated between ionizable surfaces at small separation 1511622 . Here, attaching a reservoir, we examine how $\Pi_{d}$ and $\alpha$ depend on $H$ and $\sigma_{m}$ with $\mathrm{NaOH}$ added at a density $n_{0}$. We consider the Stern layers, so we define the effective cell thickness by

$$
H^{\prime}=H-\left(d_{0}+d_{H}\right),
$$

where $d_{0}$ and $d_{H}$ are of order $5 \AA$. There is a sizable range of $H^{\prime}<\kappa^{-1}$ for not large $n_{0}$. Using Eqs.(11) and (40), we integrated the $\mathrm{PB}$ equation in the region $0<z-d_{0}<H^{\prime}$ for each given $n_{0}$ and $\sigma_{m}$. The parameters are those for silica oxide surfaces in Figs.11-16, but those for carboxylbearing surfaces are also used in Fig.17. For simplicity, we neglect the van der Waals interaction ${ }^{1 / 3}$.

\section{A. Results without applied field $(s=0)$}

First, we assume no applied field $(s=0)$. Because $d U / d z=0$ at $z=H-d_{H}$, our $H^{\prime}$ corresponds to a half of the cell thickness in the symmetric case ${ }^{15 \mid 22}$. For thick cells with $H^{\prime}>\kappa^{-1}$, it follows a well-known result 128/47,

$$
\Pi_{d} \cong 64 T n_{\mathrm{OH}}^{0} \exp \left(-2 \kappa H^{\prime}\right) .
$$

Ninham and Parsegian ${ }^{15}$ derived the power-law behavior $\Pi_{d} \propto H^{-1 / 2}$ as $H \rightarrow 0$ between symmetric ionizable walls. From analysis in Appendix E, their asymptotic behavior appears for $H^{\prime} \ll \ell_{\mathrm{GC}}$ in the case $A_{1}^{-1} \ll \alpha \ll 1$. Here, $\ell_{\mathrm{GC}}=\left(\kappa A_{1} \alpha\right)^{-1}$ is the Gouy-Chapman length in Eq. (56) shorter than $\kappa^{-1}$ in the nonlinear PB regime. The normalized potential values $U_{0}=U\left(d_{0}\right)$ and $U_{H}=$ $U\left(H-d_{H}\right)$ at the two ends are given by

$$
\begin{aligned}
U_{H}-U_{0} & \cong A_{1} \alpha \kappa H^{\prime}=H^{\prime} / \ell_{\mathrm{GC}} \ll 1, \\
\exp \left(-U_{0}\right) & \cong 4 A_{1} \alpha / \kappa H^{\prime} \gg 1 .
\end{aligned}
$$

From Eq.(11) and (46), $\Pi_{d}$ and $\alpha$ behave as

$$
\begin{aligned}
\Pi_{d} & \cong T n_{\mathrm{OH}}^{0} e^{-U_{H}} \cong T n_{\mathrm{OH}}^{0}\left(\Gamma_{0} K_{s} / K_{\mathrm{w}} H^{\prime}\right)^{1 / 2}, \\
\alpha & \cong\left(K_{s} H^{\prime} / \Gamma_{0} K_{\mathrm{w}}\right)^{1 / 2} n_{\mathrm{OH}}^{0},
\end{aligned}
$$

where we set $n_{\mathrm{H}}^{0}=K_{\mathrm{w}} / n_{\mathrm{OH}}^{0}$. With other added ions, however, Eqs.(73) and (74) should be changed appropriately (see the comments below Eqs.(42) and (46) $)^{15 / 22}$. In addition, the inequality $\alpha \ll 1$ holds for

$$
H^{\prime} \ll\left(\Gamma_{0} / K_{s}\right)\left(K_{\mathrm{w}}^{1 / 2} / n_{\mathrm{OH}}^{0}\right)^{2} .
$$



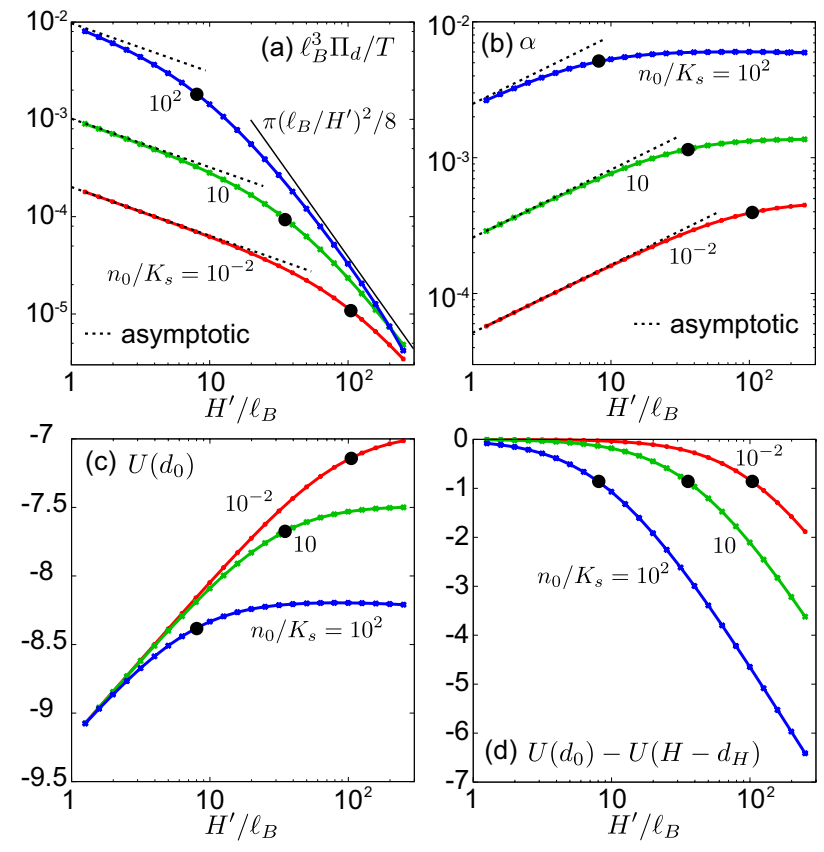

FIG. 11: Results for silica in the range $1<H^{\prime} / \ell_{B}<300$ $\left(\ell_{B}=7 \AA\right)$ without applied field $(s=0)$ for $n_{0} / K_{s}=$ $10^{-2}, 10$, and $10^{2}$. Plotted are (a) $\ell_{B}^{3} \Pi_{d} / T$, (b) $\alpha$, (c) $U\left(d_{0}\right)$, and (d) $U\left(d_{0}\right)-U\left(H-d_{H}\right)$. Points at which $H^{\prime}=\ell_{\mathrm{GC}}$ are marked by $\bullet$ on each curve. In (a) and (b), formulae (73), (74), and (78) are written as guides of eyes. In (a)-(d), Eqs.(71)(75) hold for $H^{\prime} \ll \ell_{\mathrm{GC}}$ and Eqs.(76)-(78) for $H^{\prime} \gg \ell_{\mathrm{GC}}$.

For silica oxide surfaces, we have $\Gamma_{0} / K_{s} \sim 10^{8} \mathrm{~nm}$, so Eq.(76) can well be satisfied together with Eq.(71).

On the other hand, when $\ell_{\mathrm{GC}} \ll H^{\prime} \ll \kappa^{-1}$ in the nonlinear PB regime, we find another regime (the GouyChapman regime ${ }^{28}$ ), where $U_{0}$ and $\alpha$ remain nearly at constants but $U_{H}$ strongly depends on $H^{\prime}$. That is,

$$
\begin{aligned}
& \exp \left(-U_{0}\right) \cong\left(2 A_{1} \alpha\right)^{2} \\
& \exp \left(-U_{H}\right) \cong\left(\pi / \kappa H^{\prime}\right)^{2} .
\end{aligned}
$$

Thus, $e^{U_{H}-U_{0}} \sim\left(H^{\prime} / \ell_{\mathrm{GC}}\right)^{2} \gg 1$. The $\Pi_{d}$ behaves as 28

$$
\Pi_{d} \cong T n_{\mathrm{OH}}^{0} e^{-U_{H}} \cong \pi T /\left[2 \ell_{B}\left(2 H^{\prime}\right)^{2}\right],
$$

which is independent of $n_{0}$ and $\alpha$. In the previous theories ${ }^{15 / 22}$, the behavior (78) was not found, because they adopted parameters yielding $\ell_{\mathrm{GC}} \gtrsim \kappa^{-1}$ with a salt added (see the last pragraph of Sec.IIIA).

In Fig.11, we show (a) $\Pi_{d}$, (b) $\alpha$, (c) $U_{0}$, and (d) $U_{0}-$ $U_{H}$ as functions of $H^{\prime}$ in the range $\ell_{B}<H^{\prime}<300 \ell_{B}<$ $\kappa^{-1}$ without applied field $(s=0)$ for silica oxide surfaces. We set $n_{0} / K_{s}=10^{-2}, 10$, and $10^{2}$, where $\kappa \ell_{B} \times 10^{3}$ is $0.72,1.6$, and 5.0 and $A_{1} \times 10^{-3}$ is 34,15 , and 4.9 , respectively. In these cases, $A_{1} \alpha$ remains considerably larger than 1 for $H^{\prime}>\ell_{B}=7 \AA$ from Eq.(63). We can clearly see the crossover between the two power-law regimes at $H^{\prime}=\ell_{\mathrm{GC}}$ as follows. In (a), $\Pi_{d}$ behaves as in Eqs.(73) and (78). In (b), $\alpha$ decays as $\sqrt{H^{\prime}}$ for small (a) Silica: $H^{\prime} / \ell_{B}=200$

(b) Silica: $H^{\prime} / \ell_{B}=3.3$

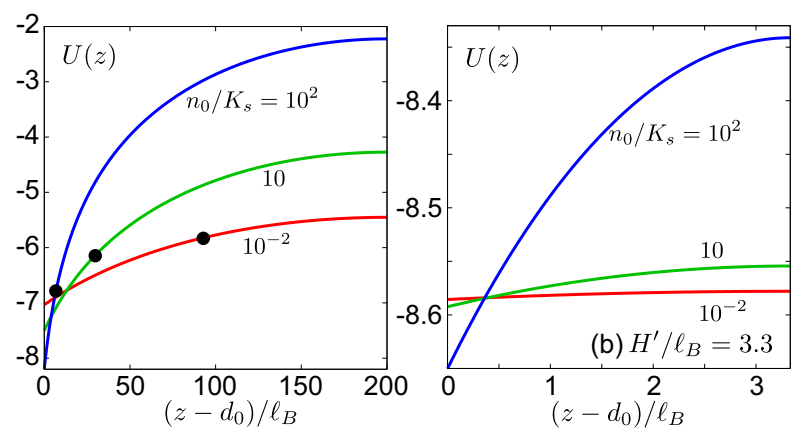

FIG. 12: Profiles of $U(z)$ in the range $0<z-d_{0}<H^{\prime}$ for $s=0$, where a silica oxide surface is at $z=0$. For $n_{0} / K_{s}=$ $10^{-2}, 10$, and $10^{2}, H^{\prime}$ is (a) $200 \ell_{B}$ with $\ell_{\mathrm{GC}}<H^{\prime}<\kappa^{-1}$ and is (b) $3.3 \ell_{B}$ with $H^{\prime}<\ell_{\mathrm{GC}}$.

$H^{\prime}$ and is a small constant for larger $H^{\prime}$. In (c), $U_{0}$ satisfies Eqs.(72) and (76) in the range $[-9,-7]$, tending to be independent of $n_{0}$ for $H^{\prime} \ll \ell_{\mathrm{GC}}$. In (d), $U_{0}-U_{H}$ behaves in accord with Eqs.(71) and (77).

In Fig.12, we display $U(z)$ without applied field for $n_{0} / K_{s}=10^{-2}, 10$, and $10^{2}$. In (a), we set $H^{\prime}=200 \ell_{B}<$ $\kappa^{-1}$; then, $H^{\prime}>\ell_{\mathrm{GC}}$ with $\ell_{\mathrm{GC}} / \ell_{B}=6.7,30$, and 93 , respectively. In (b), we set $H^{\prime}=3.3 \ell_{B}$ to realize $H^{\prime}<$ $\ell_{\mathrm{GC}}$ with $\ell_{\mathrm{GC}} / \ell_{B}=440,87$, and 10 , where the diffuse layers at the bottom and top walls largely overlap,

\section{B. Results with applied field}

Next, we apply electric field by varying $s=\sigma_{m} / e \Gamma_{0}$ for $\kappa H^{\prime}<1$. In Fig.13, $\Pi_{d}$ is displayed as a function of $H^{\prime}$ and $s$. We fix $n_{0} / K_{s}$ at 10 and $10^{3}$, where $A_{1}$ is $1.5 \times 10^{4}$ and $1.5 \times 10^{3}$, respectively. The $\Pi_{d}$ is positive around the line of $s=0$ but is mostly negative. This is natural because the surface charge densities at the two ends have the same sign only for $0<s<\alpha$. If $\kappa H^{\prime} \ll 1$, we find $\frac{53}{5}$ that $\Pi_{d}>0$ holds only for $|s| \lesssim \alpha$, where $\alpha$ is the degree of ionization at $s=0$. This width of $s$ is of order $\alpha \sim 10^{-3}$ in (a) and $10^{-2}$ in (b) from Eq.(63). In addition, for not small $|s|, \Pi_{d}$ drops to negative values with large amplitude with decreasing $H^{\prime}$ due to partial screening (see below).

In Fig.14, we plot $\alpha$ and $u=\alpha-s$ for $1<H^{\prime} / \ell_{B}<10^{2}$ and $-0.2<s<1.2$, where $n_{0} / K_{s}=10$ (left) and $10^{3}$ (right). For $H^{\prime} \gtrsim 5 \ell_{B}, \alpha$ is nearly independent of $H^{\prime}$ in the upper panels and $u$ is very small for $0<s \lesssim 1$ in the bottom panels. Thus, the self-regulation (found for $H^{\prime} \gg \kappa^{-1}$ in Sec.III) is operative even for small $H^{\prime}(\gtrsim$ $\left.5 \ell_{B}\right)$. We also write the lines of $u=0$ and $\pm\left(A_{1} H^{\prime} \kappa\right)^{-1}$. We have $H^{\prime}>\ell_{\mathrm{GC}}$ outside them. These three lines are closely located because of large $A_{1}$, which is $1.5 \times 10^{4}$ (left) and $1.5 \times 10^{3}$ (right). Indeed, on the flat area in the left, we have $-0.01<u<-0.001$ and $A_{1}|u|>15$.

In Fig.15, we show typical profiles of $U$ for $u<0$ and $s>0$ with $H^{\prime}=5 \ell_{B}=0.08 / \kappa$, which exhibit a negative 
(a) Silica: $n_{0} / K_{s}=10$

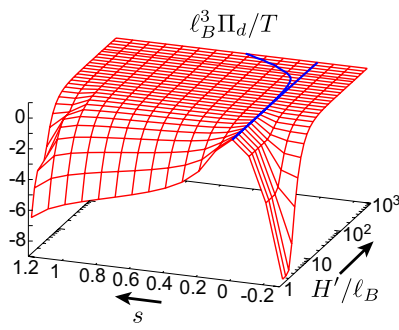

(b) Silica: $n_{0} / K_{s}=10^{3}$

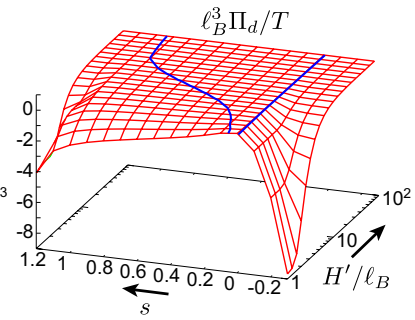

FIG. 13: $\quad \ell_{B}^{3} \Pi_{d} / T$ in the $H^{\prime} / \ell_{B^{-s}}$ plane for small $H^{\prime}$ (silica), where $n_{0} / K_{s}$ is (a) 10 and (b) $10^{3}$. Lines of $\Pi_{d}=0$ (blue) are written on the surfaces. Area of $\Pi_{d}>0$ between the two lines becomes narrower as $H^{\prime}$ is decreased ${ }^{\sqrt{53}}$ but is widened as $n_{0}$ is incresaed. For relatively large $s, \Pi_{d}$ drops to negative values with large amplitude due to partial screening. (a) Silica: $n_{0} / K_{s}=10$
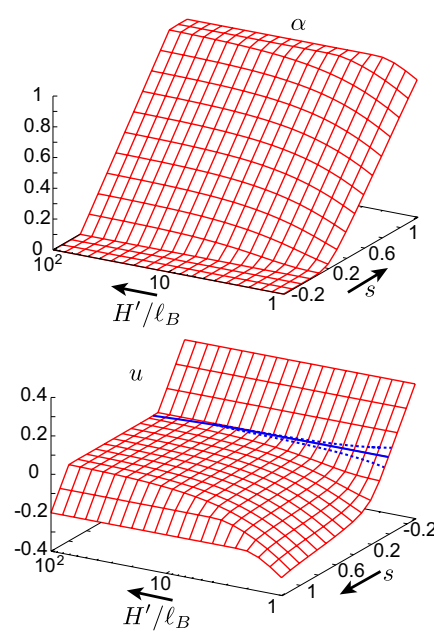

(b) Silica: $n_{0} / K_{s}=10^{3}$
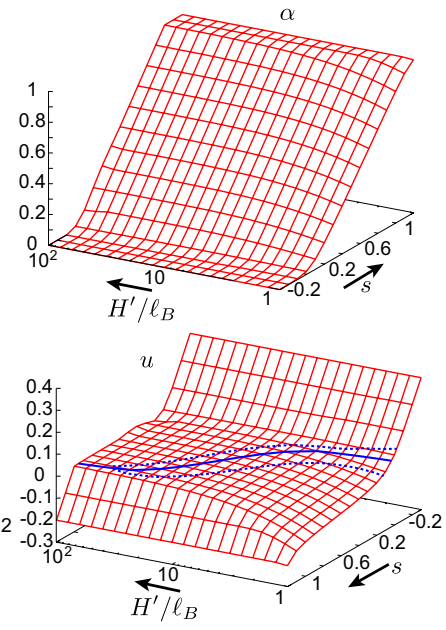

FIG. 14: $\alpha$ (top) and $u=\alpha-s$ (bottom) in the $H^{\prime} / \ell_{B}-s$ plane for silica, where $n_{0} / K_{s}$ is (a) 10 (left) and (b) $10^{3}$ (right). For not small $s(>0), \alpha$ is insensitive to $H^{\prime}$ larger than $5 \ell_{B}$. Lines of $u=\left(A_{1} H^{\prime}\right)^{-1}>0, u=0$, and $u=-\left(A_{1} H^{\prime}\right)^{-1}<0$ (from above) are written on the surfaces of $u$ (blue lines). Below (above) the middle blue line $(u=0), u$ is negative (positive).

slope far from the walls. Such profiles can appear for $\Pi_{d}<0$, so we define a dimensionless number $b$ by

$$
b=\left(-\Pi_{d} / 2 \pi T \ell_{B}\right)^{1 / 2} / \Gamma_{0},
$$

Then, $\Pi_{d} / T n_{\mathrm{OH}}^{0}=-4 A_{1}^{2} b^{2}$. The slope of $U$ is $-2 A_{1} b \kappa$ and the corresponding electric field is $4 \pi e \Gamma_{0} b / \varepsilon_{0}$ far from the walls. Here, the anions $\left(\mathrm{OH}^{-}\right)$are accumulated at the bottom and the cations $\left(\mathrm{M}^{+}\right.$and $\left.\mathrm{H}^{+}\right)$at the top, but their screening of the surface charges is only partial. The accumulated ion numbers $N_{i}=\int d z n_{i}(z)$ satisfy

$$
\begin{aligned}
& N_{\mathrm{OH}}=\Gamma_{0}(|u|-b), \\
& N_{H}+N_{M}=\Gamma_{0}(s-b),
\end{aligned}
$$

which are consistent with Eq.(12). The degree of screening (screening fraction) is given by $1-b /|u|$ at the bottom
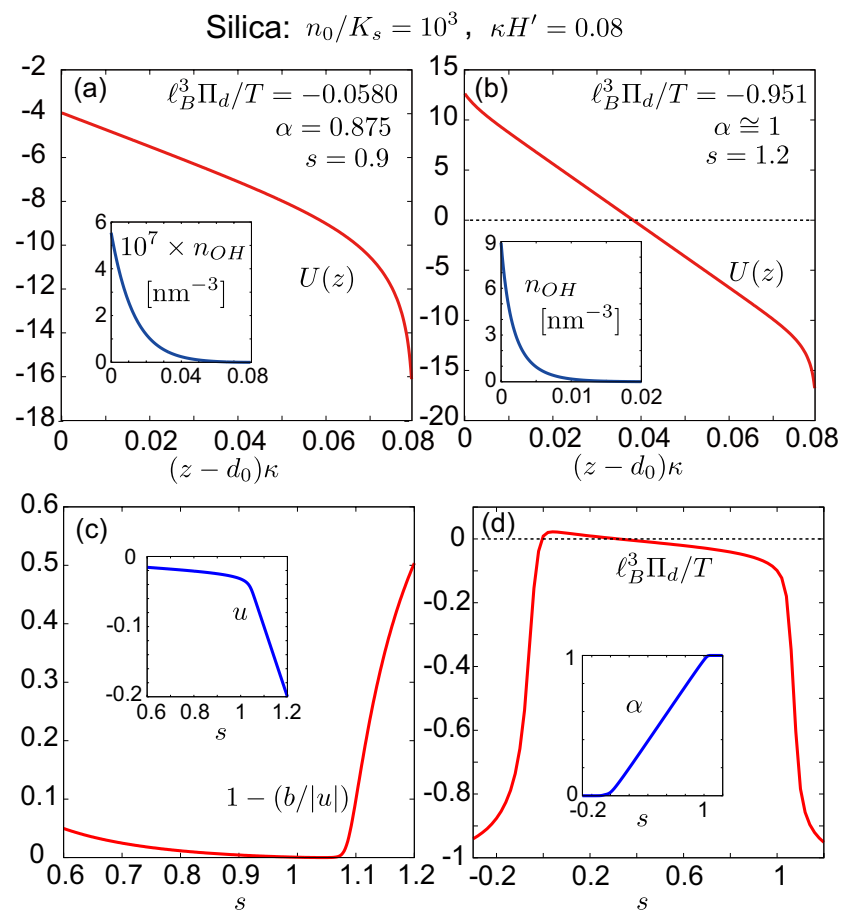

FIG. 15: Results for silica with $\kappa H^{\prime}=0.08$ and $n_{0} / K_{s}=10^{3}$, where $\kappa=2.2 \times 10^{-2} / \mathrm{nm}$. Displayed is $U(z)$ in the range $0<z-d_{0}<H^{\prime}$ for (a) $s=0.9, \alpha=0.875$, and $\ell_{B}^{3} \Pi_{d} / T=$ -0.0580 and (b) $s=1.2, \alpha=1.0$, and $\ell_{B}^{3} \Pi_{d} / T=-0.951$. Plotted also is $n_{\mathrm{OH}}(z)=n_{\mathrm{OH}}^{0} e^{U(z)}$ near the bottom in units of $\mathrm{nm}^{-3}$ (inset). (c) Screening fraction $1-b /|u|$ and $u$ (inset) vs $s$. Screening is very weak for $s<1.1$. (d) $\ell_{B}^{3} \Pi_{d} / T$ and $\alpha$ (inset) vs $s$, where $\Pi_{d}<0$ for most $s$.

and by $1-b / s$ at the top. It is 0 with no screening (with no diffuse layer) and is 1 for complete screening.

In Fig. 15 , at $n_{0} / K_{s}=10^{3}$, we find $s=0.9, \alpha=0.875$, and $b=0.0245=37 / A_{1}$ in (a) and $s=1.2, \alpha=1.0$, and $b=0.099=150 / A_{1}$ in (b). Thus, $1-b /|u|$ is 0.02 in (a) and 0.5 in (b). In fact, $n_{\mathrm{OH}}(z)$ is very small in (a) but is appreciable in (b) (inset). In (c), we show $1-b /|u|$ vs $s$ in the range $0.6<s<1.2$, which is very small for $s<1.1$ but grows abruptly for $s>1.1$. For $s<1.1$, there is almost no screening at the bottom and the screening fraction at the top is $\alpha / s$. In (d), we plot $\Pi_{d}$ and $\alpha$ in the range $-0.2<s<1.2$, In accord with Fig.13, $\Pi_{d}$ is positive only in a narrow range of $s\left(\lesssim \alpha \sim 10^{-2}\right)$. Here $u$ is negative and $H^{\prime}$ is small, but self-regulation behavior $|u| \ll 1$ in the range $0<s<1$ still persists.

The region of $\Pi_{d}>0$ is widened with increasing $n_{0}$. See the two panels in Fig.13, where $n_{0} / K_{s}=10$ and $10^{3}$. In Fig.16, this is more apparent for $n_{0} / K_{s}=10^{4}$ with $H^{\prime}=5 \ell_{B}=0.25 \kappa^{-1}$, where $n_{\mathrm{M}}^{0} \cong n_{\mathrm{OH}}^{0} \gg n_{\mathrm{H}}^{0}$. In (a), $\Pi_{d}$ is about $0.05 T \ell_{B}^{-3} \sim 250 T n_{\mathrm{OH}}^{0}$ for $0<s<$ 1 , where $U(z)$ is negative with a maximum about -6.0 and the ions in the cell are mostly $\mathrm{M}^{+}$. However, $\Pi_{d}$ becomes negative abruptly for $s>1$. In (b), the profile of $U(z)$ exhibits a changeover across $s=1$ at this $n_{0}$. For $s=0.4, E$ vanishes at the maximum point. However, 


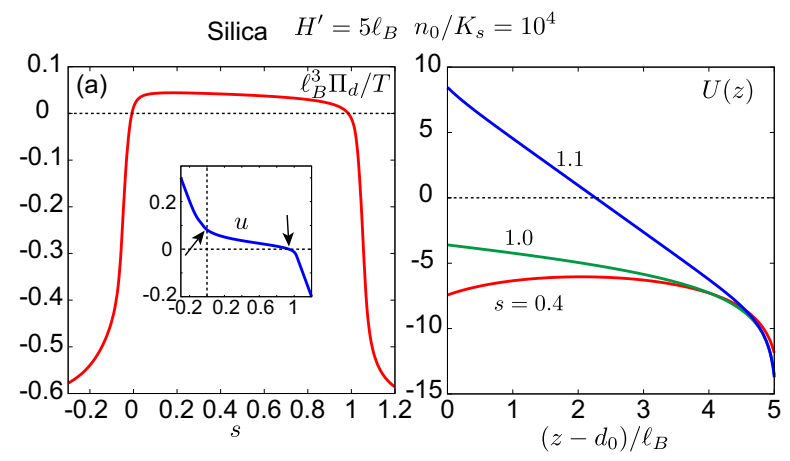

FIG. 16: (a) $\ell_{B}^{3} \Pi_{d} / T$ vs $s$ in a cell with $H^{\prime}=5 \ell_{B}=0.25 \kappa^{-1}$ for $n_{0} / K_{s}=10^{4}$. For $0<s<1$, it is positive and is of order 0.05 with small positive $u=\alpha-s$ (inset). Point of $\Pi_{d}=0$ are marked by arrows. (b) Profiles of $U(z)$ for $s=0.4,1$, and 1.1 .

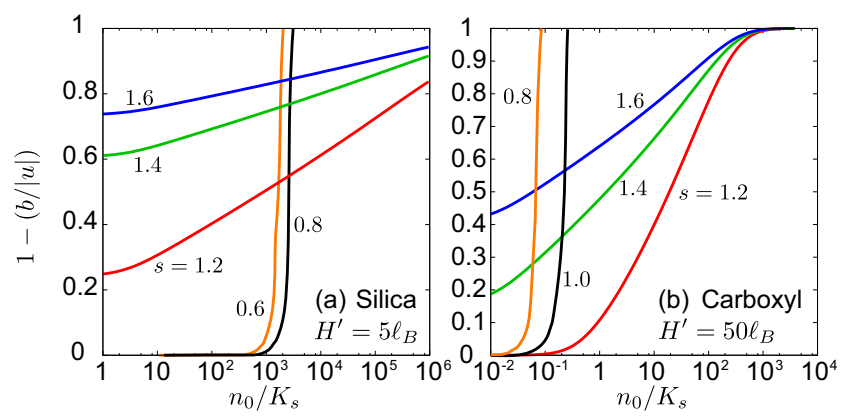

FIG. 17: Screening fraction $1-b /|u|$ vs $n_{0} / K_{s}$ at the bottom for (a) silica oxide surfaces with $H^{\prime}=5 \ell_{B}$ and (b) carboxyl-bearing surfaces with $H^{\prime}=50 \ell_{B}$ for five values of $s=\sigma_{m} / e \Gamma_{0}$. Screening increases with increasing $n_{0}$ and/or $s$. Changeover from unscreeing to screening is gradual for $s>1$ but is abrupt for $0<s<1$.

once $\Pi_{d}<0$, unscreening is significant at the bottom; in fact, $(u, 1-b /|u|)=(-0.012,0.10)$ for $s=1.0$ and $(-0.10,0.24)$ for $s=1.1$. The charcteristic features of these behaviors are unchanged even for $n_{0} / K_{s}=10^{5}$.

The screeing should be easier for larger $n_{0}$. This is confirmed in Fig.17, where plotted is $1-b /|u|$ vs $n_{0} / K_{s}$ at fixed $s$ for (a) silica oxide surfaces and (b) carboxylbearing surfaces. Here, $H^{\prime}$ is $5 \ell_{B}$ in (a) and is $50 \ell_{B}$ in (b). The $K_{s}$ and $\Gamma_{0}$ in the two systems are very different in Eqs.(15) and (67). Nevertheless, we can see significant unscreening at relatively small $n_{0}$ in the two systems. In addition, the changeover from unscreening to screening with increasing $n_{0}$ is nearly discontinuous for $0<s<1$ (from $b \cong s-\alpha$ to $b \cong 0$ ), while it is gradual for $s>1$.

\section{Theory of partial screening in thin cells}

We finally present some analytic results on the partial screening. See Appendix E for their derivations. With $b \gg A_{1}^{-1}$, we introduce a characteristic length $\ell_{\infty}$ by

$$
\ell_{\infty}=\left(2 A_{1} b\right)^{-1} \kappa^{-1}=\left(4 \pi \ell_{B} \Gamma_{0} b\right)^{-1},
$$

which is of the same order as $\ell_{\mathrm{GC}}$ in Eq.(56) for $|u| \sim b$. If we assume $U\left(H-d_{H}\right)=U_{H}<0$ and $\left|U_{H}\right| \gg 1, U(z)$ near the top is well approximated by

$$
U(z) \cong U_{H}+w+2 \ln \left(1+a_{H}-a_{H} e^{-w}\right) \quad(\text { top }),
$$

where $w=\left(H-d_{H}-z\right) / \ell_{\infty}$ and $a_{H}=s / 2 b-1 / 2$. The last term is zero at $w=0$ and tends to $2 \ln \left(1+a_{H}\right)$ for $w \gg 1$, so the profile changes on the scale of $\ell_{\infty}$. This formula excellently describes the profiles in (a) and (b) of Fig. 15 and those in Fig.16b near the top. The interior electric field is a constant for $\ell_{\infty} \ll H^{\prime}$. For the profiles in Fig.15a and those of $s=1$ and 1.1 in Fig.16b, we have $b \cong|u|$, so Eq.(83) can be used even at the bottom.

For the profile in Fig.15b, the diffusive layer near the bottom gives $b \cong 0.5|u|$. In this partial screening, the profile at the bottom is well approximated by

$$
U(z) \cong U_{0}-s-2 \ln \left(1+a_{0}-a_{0} e^{-s}\right) \quad \text { (bottom) },
$$

where $U\left(d_{0}\right)=U_{0} \gg 1, s=\left(z-d_{0}\right) / \ell_{\infty}$, and $a_{0}=$ $|u| / 2 b-1 / 2$. For the profile in Fig.15b, Eqs.(83) and (84) should coincide far from the walls, so we require

$$
\begin{gathered}
H^{\prime} / \ell_{\infty} \cong U_{0}-U_{H}-2 \ln \left[\left(1+a_{0}\right)\left(1+a_{H}\right)\right] \\
\cong 4 \ln \left(4 A_{1} b\right)+\ln \left[\frac{(|u|-b)(s-b)}{(|u|+b)(s+b)}\right]
\end{gathered}
$$

The right hand side can much exceed 1 for $A_{1} b \gg 1$. For (b) in Fig.15, Eq.(85) is nearly exact.

\section{SUMMARY AND REMARKS}

We have examined ionization on a dielectric film in water in applied field. In the geometry in Fig.1, the surface can be negatively charged with proton desorption. From the mass action law, the degree of dissociation $\alpha$ is determined by the ratio of the proton density close to the film $n_{\mathrm{H}}(0)$ and the dissociation constant $K_{s}\left(=10^{-\mathrm{pK}} \mathrm{mol} / \mathrm{L}\right)$. We have added $\mathrm{NaOH}$ at a density $n_{0}$ in water ${ }^{26}$ to decrease $n_{\mathrm{H}}(0)$. Main results are summarized as follows.

(i) In Sec.II, we have presented the free energy $F$, depending on the solute densities $n_{i}$ and the degree of ionization $\alpha$, where the the electrode charge density $\sigma_{m}$ or the potential difference $V$ is fixed. The contributions from the ionizable film and the Stern layers have been included. Minimization of the corresponding grand potential $\Omega$ yields equilibrium conditions including the mass action laws. The derivative of the equilibrium $\Omega$ with respect to the cell width $H$ yields the osmotic pressure $\Pi$.

(ii) In Sec.III, we have assumed $H \gg \kappa^{-1}$. Analysis has been made on silica oxide films with $\mathrm{pK}=7.3$ in the nonlinear PB regime, where the Gouy-Chapman length $\ell_{\mathrm{GC}}$ is shorter than $\kappa^{-1}$. We have obtained a simple 
equation for $\alpha$ in Eq.(61) for given $\sigma_{m}$ and $n_{0}$. As in Fig.7b, we have found remarkable self-regulation behavior $\alpha \cong s=\sigma_{m} / e \Gamma_{0}$ for $0<s<1$ and $n_{0} \ll n_{c}$, where $\Gamma_{0}$ is the areal density of the ionizable groups and $n_{c}$ is a crossover density. From Eq.(62), we find $n_{c}=10^{-2}$ $\mathrm{mol} / \mathrm{L}$ for silica oxide surfaces.

(iii) In Sec.IV, we have presented results using the parameters of carboxyl-bearing surfaces with $\mathrm{pK}=4.9$. General trends are common to those for slica oxcide surfaces as in Figs. 8 and 9, but numerical factors are very different. For example, we have $n_{c}=2 \times 10^{-5} \mathrm{~mol} / \mathrm{L}$.

(iv) In Sec.V, we have presented analysis for small $H^{\prime}=H-\left(d_{0}+d_{H}\right)$ with a reservoir attached. Without applied field, the disjoining pressure $\Pi_{d}$ is proportional to $\sqrt{H^{\prime}}$ for $H^{\prime}<\ell_{\mathrm{GC}}$ and to $\left(H^{\prime}\right)^{-2}$ for $\ell_{\mathrm{GC}}<H^{\prime}<\kappa^{-1}$. In applied field, the self-regulation $(\alpha \cong s$ for $0<s<1$ ) holds even for $\ell_{B}<H^{\prime}<\kappa^{-1}$. We have also found that the surface charges can be screened only partially for not small $s$. For silica oxide srfaces, $\Pi_{d}$ is mostly negative for $n_{0} / K_{s} \lesssim 10^{3}$ in Figs. 13 and $15(\mathrm{~d})$, but it assumes a large positive value in the range $0<s<1$ for $n_{0} / K_{s}=10^{4}$ in Fig.16. Similar results follow for hydroxyl-bearing surfaces as in Fig.17. We have derived analytic expressions for the potential profiles in partial screening.

(v) In Appendix A, we have examined the experimental method of imposing the fixed charge condition. In Appendix C, we have derived the expressions for the osmotic and disjoining pressures. In Appendix D, we have derived the expressions for the surface free energy for ionizable surfaces in applied field.

We make some remarks.

(1) There are a variety of ionizable surfaces with very different parameters $\left(K_{s} \text { and } \Gamma_{0}\right)^{14 \mid 17+21}$ under strong influence of ions. We have examined dissociation with small $K_{s}$, where the autoionization of water comes into play. The charge regulation has been controlled by the amount of $\mathrm{NaOH}$. If $K_{s}$ is much larger, we may add $\mathrm{HCl}$ to increase the bulk proton density $n_{\mathrm{H}}^{0}$. We can also add $\mathrm{KCl}$ to increase $\kappa$, as discussed below Eqs.(42), (46), and (74).

(2) In future we should study dynamics of surface ionization in nonstationary electric field. We note that the deprotonation on a silica-water interface takes place very slowly as rare thermal activations 54 .

(3) There are a number of nonequilibrium situations with chemical reactions ${ }^{27 / 55}$, where phase changes can take place. For example, elelectrowetting 49150 has been studied without chemical reactions.

\section{Acknowledgments}

This work was supported by KAKENHI No.25610122. RO acknowledges support from the Grant-in-Aid for Scientific Research on Innovative Areas Fluctuation and Structure (Grant No. 25103010) from the Ministry of Education, Culture, Sports, Science, and Technology of Japan. We would like to thank Junpei Yamanaka for valuable discussions.

\section{Appendix A: Realization of constant charge boundary condition}

In Fig.1, a battery has been used to produce an equilibrium electrolyte state at a given potential difference $V$. If it is disconnected, the surface charge density $\sigma_{m}$ (at the lower metal surface) becomes fixed.

As illustrated in Fig.1, we further connect a small external condenser with a capacitance $\mathcal{C}_{\text {ex }}$ to the electrodes. The potential difference of the capacitor is given by

$$
V_{\mathrm{ex}}=Q_{\mathrm{ex}} / \mathcal{C}_{\mathrm{ex}}
$$

where $Q_{\text {ex }}$ is the initial charge. After this connection, the surface charge density and the potential difference of the electrodes are changed as $\sigma_{m} \rightarrow \sigma_{m}+\Delta \sigma_{m}$ and $V \rightarrow V+\Delta V$. Then, the capacitor change is changed by $-S_{0} \Delta \sigma_{m}$, where $S_{0}$ is the surface area of the electrodes. If the potential equilibration is attained, we have

$$
V+\Delta V=\left[Q_{\mathrm{ex}}-S_{0} \Delta \sigma_{m}\right] / \mathcal{C}_{\mathrm{ex}}
$$

In the limit of small $\Delta \sigma_{m}$, we may set $\Delta V=\Delta \sigma_{m} / C_{\text {diff }}$, where $C_{\text {diff }}=\partial \sigma_{m} / \partial V$ is the differential capacitance of our system (per unit area). Then, Eq.(A2) yields

$$
\begin{aligned}
\Delta \sigma_{m}= & \left(V_{\mathrm{ex}}-V\right) /\left[S_{0} / \mathcal{C}_{\mathrm{ex}}+1 / C_{\mathrm{diff}}\right] \\
& \cong\left(V_{\mathrm{ex}}-V\right) \mathcal{C}_{\mathrm{ex}} / S_{0}
\end{aligned}
$$

where the second line holds for $C_{\text {ex }} \ll S_{0} C_{\text {diff }}$. Therefore, for sufficiently small $\mathcal{C}_{\text {ex }}, \Delta \sigma_{m}$ depends only on the initial $V$ of our system and remains fixed independently of the subsequent physical and chemical processes in the cell.

\section{Appendix B: Bulk ion densities in terms of $n_{\mathrm{MOH}}$}

In Eq.(40) we can assume the relation,

$$
n_{\mathrm{M}}^{0}+n_{\mathrm{H}}^{0}=n_{\mathrm{OH}}^{0},
$$

without loss of generality by shifting the origin of $U(z)$ appropriately $(U \rightarrow U+$ constant). Then, we obtain Eq.(41). From the chemical equilibrium conditions (4) and $(5), n_{\mathrm{H}}^{0}$ and $n_{\mathrm{M}}^{0}$ are expressed as

$$
n_{\mathrm{H}}^{0}=K_{\mathrm{w}} / n_{\mathrm{OH}}^{0}, \quad n_{\mathrm{M}}^{0}=K_{\mathrm{b}} n_{\mathrm{MOH}} / n_{\mathrm{OH}}^{0} .
$$

From Eqs.(B1) and (B2) $n_{\mathrm{OH}}^{0}$ is given by

$$
n_{\mathrm{OH}}^{0}=\left(K_{\mathrm{w}}+K_{\mathrm{b}} n_{\mathrm{MOH}}\right)^{1 / 2} .
$$

The density $n_{0}$ of $\mathrm{M}$ atoms in Eq.(7) is expressed as

$$
n_{0}=n_{\mathrm{MOH}}\left(1+K_{\mathrm{b}} / n_{\mathrm{OH}}^{0}\right) \text {. }
$$

For $\kappa H \gg 1, n_{i}^{0}$ coincide with those in Eqs.(6)-(8).

We can attach a reservoir with the same $n_{\mathrm{MOH}}$ as that in the cell. Then, the reservoir densities of $\mathrm{H}^{+}, \mathrm{OH}^{-}$, and $\mathrm{M}^{+}$are given by $n_{\mathrm{H}}^{0}$ and $n_{\mathrm{OH}}^{0}$, and $n_{\mathrm{M}}^{0}$ in Eqs.(B2) and (B3). The reservoir osmotic pressure is written as

$$
\Pi_{r}^{0}=T \sum_{i} n_{i}^{0}=T\left(n_{\mathrm{MOH}}+2 n_{\mathrm{OH}}^{0}\right) .
$$




\section{Appendix C: Force between parallel walls}

We suppose two equilibrium states in the geometry in Fig.1, where the cell length is $H$ in one state and $H+\delta H$ in the other slightly elongated one. The water density and the temperature are common. All the quantities are independent of the lateral coordinates $x$ and $y$. The grand potentials $\Omega$ and $\Omega^{\prime}$ per unit area are defined in the two states from Eq.(32) or Eq.(35). We calculate the difference $\delta \Omega=\Omega^{\prime}-\Omega$ for small $\delta H$.

In the elongated state, the quantities are denoted with a prime and the space coordinate is written as $z^{\prime}$, where $n_{i}^{\prime}\left(z^{\prime}\right)$ are the densities and $\alpha^{\prime}$ is the degree of ionization. We impose $\sigma_{m}^{\prime}=\sigma_{m}$ at fixed charge and $V^{\prime}=V$ at fixed potential difference. We assume a mapping relation between the positions $z^{\prime}$ and $z$ as 5154

$$
z^{\prime}=z+w(z),
$$

where $w(z)$ is a small displacement with $w(0)=0$ and $w(H)=\delta H$. We consider the deviations,

$$
\delta n_{i}=n_{i}^{\prime}\left(z^{\prime}\right)-n_{i}(z), \quad \delta \alpha=\alpha^{\prime}-\alpha .
$$

The mapping $(\mathrm{C} 1)$ yields $d / d z=\left(1+w^{\prime}\right) d / d z^{\prime}$ and $\int_{0}^{H+\delta H} d z^{\prime}=\int_{0}^{H} d z\left(1+w^{\prime}\right)$, with $w^{\prime}=d w / d z$. Then, from $\varepsilon_{0} d E^{\prime} / d z^{\prime}=4 \pi \rho^{\prime}\left(z^{\prime}\right)$, the deviation $\delta E=E^{\prime}\left(z^{\prime}\right)-$ $E(z)$ is related to $\delta \rho=\rho^{\prime}\left(z^{\prime}\right)-\rho(z)$ as

$$
\varepsilon_{0} \frac{d}{d z} \delta E=4 \pi\left(w^{\prime} \rho+\delta \rho\right)
$$

to linear order. From Eq.(23) the deviation in the electrostatic free energy, $\delta F_{e}=F_{e}^{\prime}-F_{e}$, is calculated as

$$
\begin{aligned}
\delta F_{e} & =\int d z w^{\prime}\left[\frac{\varepsilon_{0}}{8 \pi} E^{2}+\rho \Phi\right] \\
& +\int d z \Phi \delta \rho+\Phi(0) \delta \sigma_{\mathrm{A}}+V \delta \sigma_{m},
\end{aligned}
$$

where the first term arises from the cell elongation and the other terms coincide with those in Eq.(24). Similarly, the elongation contribution to $F_{b}$ in Eq.(26) is given by $\int d z w^{\prime} f_{b}$, where $f_{b}$ is the bulk free energy density in $F_{b}=$ $\int d z f_{b}$. From Eq,(32) or Eq.(35), we find

$$
\begin{aligned}
\delta \Omega & =-\int d z w^{\prime} \Pi-\left(N_{\mathrm{M}}+N_{\mathrm{MOH}}\right) \delta h_{0} \\
& =-\Pi \delta H-\left(N_{\mathrm{M}}+N_{\mathrm{MOH}}\right) \delta h_{0},
\end{aligned}
$$

where $\delta h_{0}=h_{0}^{\prime}-h_{0}$ and $\Pi$ is given in Eq.(44). Since $\Pi$ is a constant, we obtain the second line. Note that Eq.(C5) holds both at fixed $\sigma_{m}$ and fixed $V$.

The 1D theory in this appendix can be extended in various 3D situations. For example, we can calculate the solvent-mediated interaction between colloidal particles in a mixture solvent ${ }^{51}$. The 3D mapping $x_{\alpha}^{\prime}=x_{\alpha}+w_{\alpha}$ has been used in elasticity theory. Moreover, we can use it to derive the stress tensor for various fluids (including near-critical fluids, liquid crystals, and electrolytes 5 .
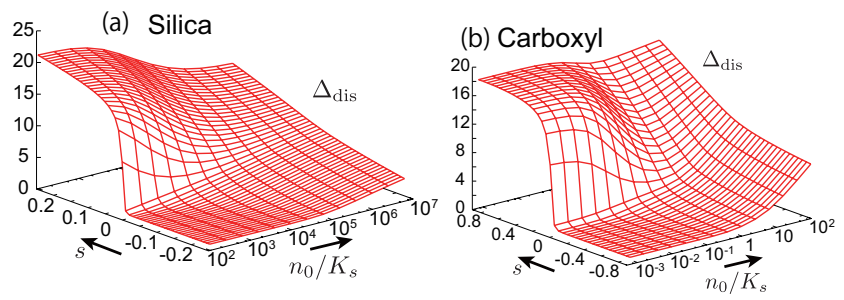

FIG. 18: Normalized decrease in the dissociation free energy per ionized group $\Delta_{\text {dis }}$ in Eq.(D5) in the $s-n_{0} / K_{s}$ plane for (a) silica and (b) carboxyl-bearing interfaces. These are calculated from the grand potential $\Omega$ in the limit $H \gg \kappa^{-1}$.

\section{Appendix D: Calculation of surface free energy}

We calculate $\Omega$ in Eq.(35) at fixed $\sigma_{m}$ and $n_{\mathrm{OH}}^{0}$ using Eqs.(18), (33), and (34). It is rewritten as

$$
\begin{aligned}
& \Omega=-\int d z\left[T \sum_{i} n_{i}+\frac{\varepsilon_{0}}{8 \pi} E^{2}\right]-\frac{\sigma_{A}^{2}}{2 C_{O}}+\frac{\sigma_{m}^{2}}{2 C_{\mathrm{tot}}}+F_{s} \\
& +T \Gamma_{0} \alpha \ln \left[n_{\mathrm{H}}(0) \lambda_{\mathrm{H}}^{3}\right]+\left[\Phi\left(d_{0}\right)-\Phi\left(H-d_{H}\right)\right] \sigma_{m}
\end{aligned}
$$

where $C_{\text {tot }}^{-1}=C_{0}^{-1}+C_{H}^{-1}+C_{d}^{-1}$. At fixed $V$, we should subtract $V \sigma_{m}$ from the right hand side.

We assume a thick cell with $\kappa H \gg 1$, where $n_{i} \rightarrow n_{i}^{0}$ and $E \rightarrow 0$ far from the walls. Then, using Eqs.(40) and (47), we can rewrite Eq.(D1) as

$$
\Omega=-H \Pi_{r}^{0}+\gamma_{H}+\gamma_{0}+\sigma_{m}^{2} / 2 C_{\text {tot }} .
$$

where $\Pi_{r}^{0}$ is the reservoir osmotic pressure in Eq.(B5). The $\gamma_{H}$ is the surface free energy from the upper diffuse layer (without surface ionization) given by

$$
\gamma_{H} / T=\left[1-\left(A_{1}^{2} s^{2}+1\right)^{1 / 2}\right] \kappa / \pi \ell_{B}+g\left(A_{1} s\right) \sigma_{m} / e,
$$

where $g(x)=2 \ln \left[\sqrt{1+x^{2}}+x\right]$. The $\gamma_{0}$ is that of the lower surface and the difference $\Delta \Omega=\gamma_{0}-\gamma_{H}$ is the contribution due to the surface ionization of the form,

$$
\begin{aligned}
& \frac{\Delta \Omega}{T \Gamma_{0}}=\frac{2}{A_{1}}\left[\left(A_{1}^{2} s^{2}+1\right)^{1 / 2}-\left(A_{1}^{2} u^{2}+1\right)^{1 / 2}\right]+\frac{F_{s}}{T \Gamma_{0}} \\
& \quad+\alpha \ln \left[n_{\mathrm{H}}(0) \lambda_{\mathrm{H}}^{3}\right]-s\left[g\left(A_{1} u\right)+g\left(A_{1} s\right)\right]-\frac{A_{2}}{2} \alpha^{2} .
\end{aligned}
$$

The above $\Delta \Omega$ coincides with $\Delta \Omega$ in Fig.5 calculated from integration of $d \Omega / d \alpha$ in Eq.(36). In fact, differentiation of Eq.(D4) with respect to $\alpha$ at fixed $s$ yields Eq.(36), since $\partial\left[\ln \left[n_{\mathrm{H}}(0)\right] / \partial \alpha=A_{2}+2 A_{1}\left(1+A_{1}^{2} u^{2}\right)^{-1 / 2}\right.$ from Eq.(59). In chemical equilibrium (11), $\alpha$ and $\Delta \Omega$ are functions of $s$ and $n_{0}$, where $F_{s} / T \Gamma_{0}+\alpha \ln \left[n_{\mathrm{H}}(0) \lambda_{\mathrm{H}}^{3}\right]=$ $\ln (1-\alpha)=-\ln \left[1+K_{s} / n_{\mathrm{H}}(0)\right]$ in Eq.(D4). We define

$$
\Delta_{\mathrm{dis}}=-\Delta \Omega / T \Gamma_{0} \alpha,
$$

in equilibrium. Then, $-T \Delta_{\text {dis }}$ is the dissociation free energy per ionized group, including the effects of the diffusive and Stern layers and the electrode. In Fig.18, we 
display $\Delta_{\text {dis }}$. For $n_{0}<n_{c}$, it is close to 1 for $\alpha \ll 1$ (for $s<0$ ) but it is 10-20 for not small $\alpha$ (for $s>0$ ). For $n_{0}>n_{c}$, it increases gradually with increasing $s$.

\section{Appendix E: Calculations for thin cells}

Calculations for $s=0$. We first present analysis in the relatively simple case of $s=0$ for $A_{1} \alpha \gg 1$, where $U(z)<0$ with large $|U|$. Since $d U / d z$ is equal to 0 at $z=H-d_{H}$ and to $2 \kappa A_{1} \alpha$ at $z=d_{0}$, Eq.(46) yields

$$
\begin{aligned}
& \kappa^{-2}(d U / d z)^{2} \cong e^{-U(z)}-e^{-U_{H}}, \\
& \left(2 A_{1} \alpha\right)^{2} \cong e^{-U_{0}}-e^{-U_{H}},
\end{aligned}
$$

We can integrate Eq.(E1) in the following form,

$$
\kappa\left(H-d_{H}-z\right) \cong 2 e^{U_{H} / 2} \tan ^{-1}(\sqrt{q(z)})
$$

where we use $\int_{0}^{x} d y\left(1 / \sqrt{e^{y}-1}\right)=2 \tan ^{-1}\left(\sqrt{e^{x}-1}\right)$ by setting $y=U_{H}-U$. We define

$$
q(z)=\exp \left[U_{H}-U(z)\right]-1 .
$$

where $q\left(H-d_{H}\right)=0$ and $q\left(d_{0}\right)=\left(2 A_{1} \alpha\right)^{2} \exp \left(U_{H}\right)$. There are two cases. If $q \ll 1$, we can set $\tan ^{-1} \sqrt{q} \cong$
$\left(U_{H}-U\right)^{1 / 2} \ll 1$ in Eq.(E3) to obtain $H^{\prime} \ll \ell_{\mathrm{GC}}$ and Eqs.(71)-(74). On the other hand, if $q \gg 1$, we can set $\tan ^{-1} \sqrt{q} \cong \pi / 2$ to obtain $H^{\prime} \gg \ell_{\mathrm{GC}}$ and Eqs.(76)-(78).

Calculations of partial screening. We explain the partial screening in Fig. 15 with $s>0$ and $u<0$. In terms of $b$ in Eq.(79), the PB equation in Eq.(46) becomes

$$
2 \cosh (U)-2=\kappa^{-2}(d U / d z)^{2}-\left(2 A_{1} b\right)^{2} .
$$

At $z=d_{0}$ and $H-d_{H}$, we find

$$
\begin{aligned}
& \cosh \left(U_{0}\right)-1=2 A_{1}^{2}\left(u^{2}-b^{2}\right), \\
& \cosh \left(U_{H}\right)-1=2 A_{1}^{2}\left(s^{2}-b^{2}\right),
\end{aligned}
$$

If $U_{H}<0$ and $\left|U_{H}\right| \gg 1$, we can replace $\cosh (U)-1$ by $e^{-U} / 2$ in Eq.(E5) near the top. It follows Eq.(83). Integration of $n_{\mathrm{H}}(z) / n_{\mathrm{H}}^{0}=n_{\mathrm{M}}(z) / n_{\mathrm{M}}^{0}=e^{-U}$ near the top can be performed to give Eq.(81). On the other hand, if $U_{0} \gg 1$, we can replace $\cosh (U)-1$ by $e^{U} / 2$ near the bottom. Then, Eq.(84) is obtained and integration of $n_{\mathrm{OH}}(z) / n_{\mathrm{OH}}^{0}=e^{U}$ near the bottom yields Eq.(80).
1 J. N. Israelachvili, Intermolecular and Surface Forces (Academic Press, London, 1991).

2 R. J. Hunter, Foundations of Colloid Science (Oxford University Press, Oxford, 2001). See Chap.10 in this book.

${ }^{3}$ H.-J. Butt, K. Graf, and M. Kappl, Physics and Chemistry of Interfaces, 3rd ed. (Wiley-VCH Verlag GmbH, Weinheim, 2013).

4 D. Ben-Yaakov, D. Andelman, R. Podgornik, D. Harries, Current Opinion in Colloid and Interface Science 16, 542 (2011).

5 T. W. Healy and D. W. Fuerstenau, J. Colloid Sci. 20, 376 (1965).

6 J. Westall and H. Hohl, Adv. Colloid Interface Sci. 12, 265 (1980).

7 T. Hiemstra, J. C. M. de Wit, and W. H. van Riemsdijk, J. Colloid Interface Sci. 133, 105 (1989).

${ }^{8}$ K. F. Hayes, G. Redden, W. Ela, and J. O. Leckie, J. Colloid and Interf. Sci. 142, 448 (1991).

9 J. J. Sonnefeld, J. Colloid Interface Sci. 155, 191 (1993).

10 R.E.G. van Hal, J.C.T. Eijkel, and P. Bergveld, Adv. Colloid Interface Sci.69, 31 (1996).

11 J. N. Israelachvili and R. M. Pashley, J. of Colloid and Interface Science, 98, 500 (1984).

12 A. Grabbe, Langmuir 9, 797 (1993).

13 C. Zhao, D. Ebeling, I. Siretanu, D. van den Ende, and F. Mugele, Nanoscale 7, 16298 (2015).

14 G. Trefalt, S. H. Behrens, and M. Borkovec, Langmuir 32, 380 (2016).

15 B. W. Ninham and V. A. Parsegian, J. Theor. Biol. 31, 405428 (1971).

16 D. Y. C. Chan, T. W. Healy, and L. R. White, J. Chem. Soc., Faraday Trans. I, 172, 2844 (1976).

17 S. H. Behrens and M. Borkovec, J. Phys. Chem. B 103,
2918 (1999); Phys. Rev. E 60, 7040 (1999).

18 S. H. Behrens, D. Iso Christl, R. Emmerzael, P. Schurtenberger, and M. Borkovec Langmuir 16, 2566 (2000).

19 S. H. Behrens and D.G. Grier, J. Chem. Phys. 2001, 115, 6716 (2001).

${ }^{20}$ P. W. Biesheuvel and W. B. S. de Lint, J. Colloid Interface Sci. 241, 422 (2001); P. M. Biesheuvel, Langmuir 17, 3553 (2001).

21 M. Heinen, T. Palberg, and H. Löwen J. Chem. Phys. 140, 124904 (2014)

22 T. Markovich, D. Andelman, and R. Podgornik, EPL, 113 ,26004 (2016). Their expression for $\Pi_{d}$ in the NinhamParsegian regime is obtained if $n_{\mathrm{OH}}^{0} / n_{\mathrm{H}}^{0}=\left(n_{\mathrm{OH}}^{0}\right)^{2} / K_{\mathrm{w}}$ in our expression in Eq.(73) is replaced by 1.

23 M. Eigen and L. De Maeyer, Z. Elektrochem. 59, 986 (1955); M. Eigen and L. De Maeyer, Proc. Roy. Soc. (London), Ser. A 247, 505 (1958).

24 T. Gisler, S. F. Schulz, M. Borkovec, H. Sticher, P. Schurtenberger, B. D'Aguanno, and R. Klein, J. Chem. Phys. 101, 9924 (1994).

25 R. Pericet-Camara, G. Papastavrou, S. H. Behrens, and M. Borkovec, J. Phys. Chem. B 108, 19467 (2004).

26 J. Yamanaka, Y. Hayashi, N. Ise, and T. Yamaguchi, Phys. Rev. E 55, 3028 (1997).

27 M. Murai, H. Yamada, J. Yamanaka, S. Onda, M. Yonese, K. Ito, T. Sawada, F. Uchida, and Y. Ohki, Langmuir 23, 7510 (2007).

28 D. Andelman, Introduction to Electrostatics in Soft and Biological Matter. In Soft Condensed Matter Physics in Molecular and Cell Biology; Poon, W., Andelman, D., Eds.; Scottish Graduate Series; Taylor \& Francis: New York, 2006; p 97.

29 A. Onuki, R. Okamoto, and T. Araki, Bull. Chem. Soc. 
Jpn. 84 (2011) 284113.

30 R. Okamoto and A. Onuki, Phys. Rev. E 84, 051401 (2011)

31 E. Raphael and J. F. Joanny, Europhys. Lett. 13, 623 (1990).

32 I. Borukhov, D. Andelman, and H. Orland, Eur. Phys. J. B 5, 869 (1998).

33 Y. Burak and R. R. Netz, J. Phys. Chem. B 108, 4840 (2004)

34 R. Okamoto and A. Onuki, J. Chem. Phys. 131, 094905 (2009).

35 A. Onuki and R. Okamoto, J. Phys. Chem. B 113, 3988 (2009).

36 M. Muthukumar, J. Chem. Phys. 120, 9343 (2004); M. Muthukumar, J. Hua, and A. Kundagrami, J. Chem. Phys. 132, 084901 (2010).

37 G. S. Longo, M. O. de la Cruz, and I. Szleifer, Soft Matter, 8, 1344 (2012).

38 T. Araki, Soft Matter, 12, 6111 (2016).

${ }^{39}$ C. B. Post and B. H. Zimm, Biopolymers 21, 2139 (1982); . P. G. Arscott, C. Ma, J. R. Wenner, and V. A. Bloomfield, Biopolymers 36, 345 (1995); A. Hultgren and D. C. Rau, Biochemistry 43, 8272 (2004); C. Stanley and D. C. Rauy, Biophys. J. 91, 912 (2006).

40 D. M. Kolb, Surf. Sci., 500, 722 (2002).

41 J. Hautman, J. W. Halley, and Y.-J. Rhee, J. Chem. Phys. 91, 467 (1989).

42 I.-C. Yeh and M.L. Berkowitz, J. Chem. Phys. 111, 3155 (1999).

43 P. S. Crozier, R. L. Rowley, and D. Henderson, J. Chem. Phys. 113, 9202 (2000).
44 A. P.Willard, S. K. Reed, P. A. Madden, and D. Chandler, Faraday Discuss. 141, 423 (2009).

${ }^{45}$ K. Takae and A. Onuki, J. Phys. Chem. B 119, 9377 (2015); J. Chem. Phys. 143, 154503 (2015)

46 As the Stern potential drop, we find the expression $V_{0}^{\mathrm{S}}=$ $4 \pi \int_{0}^{d_{0}} d z\left[P_{b}-P(z)\right]$, where $P_{b}=\left(\varepsilon_{0}-1\right) E\left(d_{0}\right) / 4 \pi$ is the bulk polarization close to the bottom Stern layer 45 .

47 H. Ohshima, Theory of Colloid and Interfacial Electric Phenomena (Elsevier, New York, 2006).

48 L. D. Landau and E. M. Lifshitz, Electrodynamics of Continuous Media (Pergamon, New York, 1984), Vol. 8.

49 D. Klarman and D. Andelman, Langmuir 27, 6031 (2011).

${ }^{50}$ F. Mugele and J.C. Baret, J. Phys.: Cond. Mat. 17, R705 (2005).

51 R. Okamoto and A. Onuki, Phys. Rev. E 88, 022309 (2013).

${ }^{52}$ K. Aoki, T. Li, J. Chen, and T. Nishiumi, J. Electroanal. Chem. 613, 1 (2008); K. Aoki, T. Li, J. Chen, and T. Nishiumi, J. Electroanal. Chem. 633, 319 (2009).

53 Setting $\Pi_{d}=0$, we solve Eq.(41) for $u<0$ and $s>0$ exactly as $\tanh (U / 4)=-C \exp \left[\kappa\left(z-d_{0}\right)\right]$. For $A_{1}|u| \gg 1$ we have $C \cong 1+1 / A_{1}|u|$ and $s(|u|-\alpha) \cong \alpha / 2 A_{1} \kappa H^{\prime}$. Then, $s \cong \alpha$ for $H^{\prime}>1 / A_{1} \alpha \kappa=\ell_{\mathrm{GC}}$.

54 A. Onuki, Phase Transition Dynamics (Cambridge University Press, Cambridge, 2002). See Appendix 6A in this book.

55 M. Z. Bazant, M. S. Kilic, B. D. Storey, and A. Ajdari, Adv. Colloid Interface Sci. 152, 48 (2009). 